\title{
Well-posedness of formulations of the Einstein equations with dynamical lapse and shift conditions
}

\author{
Carsten Gundlach \\ School of Mathematics, University of Southampton, Southampton SO17 1BJ, United Kingdom \\ José M. Martín-García \\ Instituto de Estructura de la Materia, Centro de Física Miguel A. Catalán, C.S.I.C., Serrano 123, 28006 Madrid, Spain
}

(Received 8 April 2006; published 14 July 2006)

\begin{abstract}
We prove that when the equations are restricted to the principal part the standard version of the Baumgarte-Shapiro-Shibata-Nakamura (BSSN) formulation of the Einstein equations is equivalent to the Nagy-Ortiz-Reula (NOR) formulation for any gauge, and that the Kidder-Scheel-Teukolsky (KST) formulation is equivalent to NOR for a variety of gauges. We review a family of elliptic gauge conditions and the implicit parabolic and hyperbolic drivers that can be derived from them, and show how to make them symmetry-seeking. We investigate the hyperbolicity of Arnowitt-Deser-Misner (ADM), NOR, and BSSN with implicit hyperbolic lapse and shift drivers. We show that BSSN with the coordinate drivers used in recent "moving puncture" binary black hole evolutions is ill-posed at large shifts, and suggest how to make it strongly hyperbolic for arbitrary shifts. For ADM, NOR, and BSSN with elliptic and parabolic gauge conditions, which cannot be hyperbolic, we investigate a necessary condition for wellposedness of the initial-value problem.
\end{abstract}

DOI: 10.1103/PhysRevD.74.024016

PACS numbers: 04.20.Cv, 04.20.Ex, 04.25.Dm

\section{INTRODUCTION}

Numerical solutions of the Einstein equations are often obtained by specifying initial data on a hypersurface and evolving in time. The Einstein equations then split into evolution equations which contain time derivatives and constraints which do not. A choice of variables and of evolution equations for them is called a formulation of the Einstein equations. At the same time, some metric components are not determined by the evolution equations or constraints and must be determined otherwise to obtain a closed system. This is called a choice of gauge.

A standard formulation is the Arnowitt-Deser-Misner (ADM) one, whose variables are the metric $\gamma_{i j}$ and extrinsic curvature $K_{i j}$ of a spacelike hypersurface. The ADM evolution equations for these two tensors are first order in time and second order in space. The ADM constraint equations are second order in space. The $\gamma_{i j}$ represent 6 out of 10 components of the metric $g_{\mu \nu}$ of the spacetime. The other 4 components can be parametrized by a scalar $\alpha$, the lapse, and a 3 -vector $\beta^{i}$, the shift. The lapse and shift do not appear in the ADM constraints. They appear in the evolution equations, but without time derivatives. They must therefore be determined by a gauge choice.

One distinguishes fixed gauge choices, in which the lapse (or the densitized lapse) and shift are given a priori as functions of the coordinates $x^{\mu}$, and live gauge conditions. The latter can be subdivided into algebraic ones where the lapse and shift are algebraically related to the dynamical variables, differential (typically elliptic) constraints on the lapse and shift, and evolution equations for them.
In order for numerical solutions to converge to the continuum solution, given that discretization error in the evolution equations generates constraint violations, the continuum time evolution problem must be well-posed for arbitrary initial data that do not obey the constraints. In this paper we investigate the well-posedness of a number of live gauge conditions combined with the ADM formulation and three formulations that are derived from it, the Nagy-Ortiz-Reula (NOR), Baumgarte-Shapiro-ShibataNakamura (BSSN), and Kidder-Scheel-Teukolsky (KST) formulations.

In Sec. II we review the formulations while leaving the gauge still undetermined. We show that, roughly speaking, the NOR formulation is equivalent in the principal part to the BSSN formulation and the KST formulation. For the KST formulation this will require shedding three variables that evolve trivially.

In Sec. III we review live gauge conditions. Our guiding principle is that the gauge should be "symmetry-seeking." Specifying the derivatives of the lapse and shift along the normal to the time slices makes it easier to establish wellposedness analysis but specifying their time derivative makes it easier to show a gauge is symmetry-seeking. We show that the two time derivatives are equivalent in some cases.

In Secs. IV, V, and VI we combine formulations and gauges, and investigate the well-posedness of the resulting closed systems. What we can prove depends on the choice of gauge. In Secs. IV and V we investigate gauges in which the whole system is strongly or symmetric hyperbolic. This gives us both necessary and sufficient conditions for wellposedness. In Sec. VI we use mode analysis to find a 
necessary condition for the well-posedness of the Cauchy problem of the remaining, nonhyperbolic, systems. Section VII contains our conclusions.

\section{EQUIVALENCE OF FORMULATIONS OF THE EINSTEIN EQUATIONS}

\section{A. Definitions}

Well-posedness of an initial-value problem implies that an estimate

$$
\|\delta u(\cdot, t)\| \leq F(t)\|\delta u(\cdot, 0)\|
$$

exists, where $u(x, t)$ is the solution, $u(x, 0)$ the initial data, $\delta$ denotes a linear perturbation, and $\|\cdot\|$ stands for appropriate norms (which may involve spatial derivatives), and where $F(t)$ is independent of the initial data. This means that the solution depends continuously on the initial data. Necessary or sufficient conditions for the well-posedness of the initial-value problem, such as strong hyperbolicity, can usually be determined from the principal part of the evolution equations.

In this section we discuss formulations of the $3+1$ Einstein equations derived from the ADM formulation without yet specifying the lapse and shift. These are systems of evolution equations which are first order in time in all evolved variables, but second order in space in some variables $v$ and $z$ and first order in other variables $w$. We shall therefore be looking at quasilinear systems of equations whose principal part is

$$
\begin{gathered}
\dot{v} \simeq \partial v+w+\partial z, \\
\dot{w} \simeq \partial \partial v+\partial w+\partial \partial z,
\end{gathered}
$$

where $\simeq$ indicates equality in the principal part, a dot denotes $\partial_{t} \equiv \partial / \partial t$, and $\partial$ stands for spatial derivatives. We will close this system later by providing evolution or constraint equations for the (gauge) variables $z$.

We shall show that for certain parameter choices and in certain gauges these formulations are equivalent, roughly in the sense that there is a bijection between their variables and their principal parts, and that the initial-value problem for one system is well-posed if and only if it is well-posed for the other. The detailed results will be stated in a Lemma at the end of each subsection.

\section{B. ADM}

We write the spacetime metric in the well-known $3+1$ form

$$
d s^{2}=-\alpha^{2} d t^{2}+\gamma_{i j}\left(d x^{i}+\beta^{i} d t\right)\left(d x^{j}+\beta^{j} d t\right) .
$$

The ADM formulation of the Einstein equations in the form given by York [1] is

$$
\partial_{t} \gamma_{i j}=\mathcal{L}_{\beta} \gamma_{i j}-2 \alpha K_{i j},
$$

$$
\partial_{t} K_{i j}=\mathcal{L}_{\beta} K_{i j}-D_{i} D_{j} \alpha+\alpha\left(R_{i j}-2 K_{i l} K_{j}^{l}+K K_{i j}\right),
$$

$$
\begin{gathered}
H \equiv R-K_{i j} K^{i j}+K^{2} \doteq 0, \\
M_{i} \equiv D_{j} K_{i}^{j}-D_{i} K \doteq 0,
\end{gathered}
$$

where $D_{i}$ is the 3-dimensional covariant derivative compatible with $\gamma_{i j}, \mathcal{L}_{\beta}$ is the Lie derivative along $\beta^{i}$, and $R_{i j}$ is the Ricci tensor of $\gamma_{i j}$. Indices are moved implicitly with $\gamma_{i j}$ and $\gamma^{i j}$ throughout this paper. (Note the distinction between $\equiv$, which denotes the definition of a shorthand notation, and $\doteq$, which denotes a constraint or, below, the definition of an auxiliary variable.) For convenience of notation we define the shorthands

$$
d_{i} \equiv \gamma^{j k} \gamma_{i j, k}, \quad t_{i} \equiv \gamma^{j k} \gamma_{j k, i}
$$

where a comma denotes a partial derivative. With these the principal part (second derivatives of $\gamma_{i j}$ ) of $R_{i j}$ is

$$
R_{i j} \simeq-\frac{1}{2}\left(\gamma_{i j, k}, k+t_{(i, j)}\right)+d_{(i, j)} .
$$

The ADM evolution equations are first order in time in the variables $v=\gamma_{i j}$ and $w=K_{i j}$. The highest spatial derivatives in the ADM constraints and the ADM evolution equations are second spatial derivatives of $\gamma_{i j}$ and $\alpha$, and first derivatives of $K_{i j}$ and $\beta^{i}$. The principal part of the Hamiltonian and momentum constraints are

$$
\begin{gathered}
H \simeq d_{i}{ }^{i}-t_{i}{ }^{i}, \\
M_{i} \simeq K_{i j}{ }^{j}-K_{, i} .
\end{gathered}
$$

Throughout this article we consider the vacuum Einstein equations. The generalization to matter is trivial in the typical case, for example, perfect fluids or electromagnetism, where the Einstein equations and the matter equations couple only through lower-order terms.

\section{NOR}

The NOR formulation [2] is obtained from the ADM formulation by introducing the auxiliary variables

$$
f_{i} \doteq d_{i}-\frac{\rho}{2} t_{i}
$$

where $\rho$ is a constant parameter. This gives rise to the auxiliary constraints

$$
G_{i} \equiv f_{i}-d_{i}+\frac{\rho}{2} t_{i} \doteq 0 .
$$

The full NOR evolution equations are defined as

$$
\partial_{t} \gamma_{i j}=\mathrm{ADM}
$$

$$
\partial_{t} K_{i j}=\mathrm{ADM}+a \alpha G_{(i, j)},+\alpha \gamma_{i j}\left(c H+d G_{k}{ }^{, k}\right),
$$




$$
\partial_{t} f_{i}=\partial_{t}\left(d_{i}-\frac{\rho}{2} t_{i}\right)_{\mathrm{ADM}}+\mathcal{L}_{\beta} G_{i}+2 b \alpha M_{i},
$$

where ADM in Eqs. (15) and (16) represents the right-hand sides of (5) and (6), respectively, and the subscript ADM in (17) means that the time derivative of $d_{i}$ and $t_{i}$ must be replaced by their expressions obtained from Eq. (5). $a, b, c$, and $d$ are constant parameters to be fixed later. (We have replaced the parameter $a^{\prime}$ of [3] by $d \equiv c a^{\prime}$.)

In the following we use the shorthand

$$
\partial_{0} \equiv \alpha^{-1}\left(\partial_{t}-\beta^{k} \partial_{k}\right)
$$

to hide the transport terms in the principal part of the evolution equations. We also densitize the lapse as

$$
\alpha \equiv|\gamma|^{\sigma / 2} Q,
$$

where $Q$ rather than $\alpha$ is now considered as the dynamical variable. (Later we will often set $\sigma=0$, so that $Q=\alpha$. However, $\sigma>0$ is necessary to obtain a hyperbolic formulation with fixed lapse and shift.) The principal part of the NOR evolution equations is then

$$
\begin{gathered}
\partial_{0} \gamma_{i j}=-2 K_{i j}+2 \alpha^{-1} \beta_{,(i}^{k} \gamma_{j) k}, \\
\partial_{0} K_{i j} \simeq-(\ln Q)_{, i j}+a f_{(i, j)}-\frac{1}{2} \gamma_{i j, k}{ }^{, k}+(1-a) d_{(i, j)} \\
+\frac{1}{2}(a \rho-1-\sigma) t_{(i, j)}+\gamma_{i j}\left(c H+d G_{k}^{, k}\right), \\
\partial_{0} f_{i} \simeq 2(b-1) K_{i j}{ }^{j}+(\rho-2 b) K_{, i}+\alpha^{-1} \gamma_{i k} \beta^{k},{ }_{, j}{ }^{\prime} \\
+(1-\rho) \alpha^{-1} \beta^{j}{ }_{, i j} .
\end{gathered}
$$

In contrast to the ADM evolution equations, the NOR evolution equations contain second spatial derivatives of the shift in the evolution equation for $f^{i}$. We define $v=$ $\gamma_{i j}, z=\left(\ln Q, \beta^{i}\right)$, and $w=\left(K_{i j}, f_{i}\right)$. With $a=b=c=$ $d=0$, the evolution of $f_{i}$ can be ignored, and ADM is recovered as a limiting case of NOR.

The Hamiltonian constraint of the NOR system is defined only up to use of the constraint $G_{i}$. For definiteness, we denote by $H$ the ADM Hamiltonian defined in (7) in terms of $\gamma_{i j}$ and $K_{i j}$ but not $f_{i}$. The evolution equations imply a closed constraint evolution system whose principal part is

$$
\begin{aligned}
& \partial_{0} H \simeq-2 M_{i}{ }^{i}, \\
& \partial_{0} M_{i} \simeq-\left(\frac{1}{2}+2 c\right) H_{, i}+\frac{a}{2} G_{i, j}{ }^{j}-\left(\frac{a}{2}+2 d\right) G_{j, i}{ }^{j}, \\
& \partial_{0} G_{i} \simeq 2 b M_{i} .
\end{aligned}
$$

In particular, the ADM constraint evolution is given by (23) and (24) with $a=b=c=d=0$. (The variable $f_{i}$ then decouples from $\gamma_{i j}$ and $K_{i j}$ and can be neglected).

\section{BSSN}

The BSSN formulation (see for example [4]) is obtained from the ADM formulation by introducing the new variables

$$
\begin{gathered}
\tilde{\gamma}_{i j} \doteq(\operatorname{det} \gamma)^{-1 / 3} \gamma_{i j}, \\
\tilde{\Gamma}^{i} \doteq \tilde{\gamma}^{i j} \tilde{\gamma}^{k l} \tilde{\gamma}_{j k, l}, \\
\phi \equiv \frac{1}{12} \ln \operatorname{det} \gamma, \quad K \equiv \gamma^{i j} K_{i j}, \\
\tilde{A}_{i j} \doteq(\operatorname{det} \gamma)^{-1 / 3}\left(K_{i j}-\frac{1}{3} \gamma_{i j} K\right) .
\end{gathered}
$$

With a densitized lapse we then have $\ln \alpha=\ln Q+6 \sigma \phi$. The definition of the $\tilde{\Gamma}^{i}$ gives rise to the differential constraint

$$
G_{i} \equiv \tilde{\gamma}_{i j} \tilde{\Gamma}^{j}-\tilde{\gamma}^{j k} \tilde{\gamma}_{i j, k} \doteq 0 .
$$

The definition of $\tilde{A}_{i j}$ gives rise to the algebraic constraint

$$
T \equiv \tilde{\gamma}^{i j} \tilde{A}_{i j} \doteq 0
$$

and from the definition of $\tilde{\gamma}_{i j}$ we have the algebraic constraint

$$
D \equiv \ln \operatorname{det} \tilde{\gamma} \doteq 0 .
$$

(There are no definition constraints associated with the new variables $\phi$ and $K$ because $\gamma_{i j}$ and $K_{i j}$ are no longer variables.) Here we do not define the full BSSN evolution equations but only their principal part. It is

$$
\begin{gathered}
\partial_{0} \phi \simeq-\frac{1}{6} K+\frac{1}{6 \alpha} \beta_{, i}^{i}, \\
\partial_{0} \tilde{\gamma}_{i j} \simeq-2 \tilde{A}_{i j}+2 \alpha^{-1}\left(\beta^{k}{ }_{(, i} \tilde{\gamma}_{j) k}-\frac{1}{3} \tilde{\gamma}_{i j} \beta^{k}{ }_{, k}\right), \\
\partial_{0} K \simeq-\left[(\ln Q)_{, i j}+6 \sigma \phi \phi_{, i j}\right] e^{-4 \phi} \tilde{\gamma}^{i j}, \\
\partial_{0} \tilde{A}_{i j} \simeq e^{-4 \phi}\left[-(\ln Q)_{, i j}-\frac{1}{2} \tilde{\gamma}^{m n} \tilde{\gamma}_{i j, m n}-2(1+3 \sigma) \phi_{, i j}\right. \\
\left.\left.+a \tilde{\gamma}_{k(i} \tilde{\Gamma}^{k}{ }_{, j}\right)+(1-a) \tilde{\gamma}^{k l} \tilde{\gamma}_{k(i, j) l}\right]^{\mathrm{TF}}, \\
\partial_{0} \tilde{\Gamma}^{i} \simeq 2(b-1) \tilde{\gamma}^{i j} \tilde{\gamma}^{k l} \tilde{A}_{j k, l}-\frac{4}{3} b \tilde{\gamma}^{i j} K_{, j}+\alpha^{-1} \tilde{\gamma}^{j k} \beta_{, j k}^{i} \\
+\frac{1}{3} \alpha^{-1} \tilde{\gamma}^{i k} \beta^{j}{ }_{, j k},
\end{gathered}
$$

where TF indicates the tracefree part. (The entire righthand side of (36) is made tracefree, not only the principal part.) The parameters $a$ and $b$ have been introduced for comparison with NOR. In standard BSSN, $a=b=1$.

For definiteness, we define the Hamiltonian and momentum constraint for the BSSN system by substituting the inverse of the definitions (26) and (29) into the ADM definitions of these constraints. We again use $M_{i}$ and $H$ to denote the resulting expressions. They do not contain the variables $\tilde{\Gamma}^{i}$. Their principal part is 


$$
\begin{gathered}
H \simeq e^{-4 \phi} \tilde{\gamma}^{i j}\left(\tilde{\gamma}^{k l} \tilde{\gamma}_{k i, j l}-8 \phi_{, i j}\right), \\
M_{i} \simeq \tilde{A}_{i j, k} \tilde{\gamma}^{j k}-\frac{2}{3} K_{, i} .
\end{gathered}
$$

The principal part of the evolution system for these constraints is

$$
\begin{aligned}
& \partial_{0} H \simeq-2 e^{-4 \phi} \tilde{\gamma}^{i j} M_{i, j} \\
& \partial_{0} M_{i} \simeq \frac{1}{6} H_{, i}+e^{-4 \phi}\left(\frac{a}{2} \tilde{\gamma}^{j k} G_{i, j k}+\frac{a}{6} \tilde{\gamma}^{j k} G_{j, i k}+\frac{1}{6} \tilde{\gamma}^{j k} D_{, i j k}\right) \\
& \partial_{0} G_{i} \simeq 2 b M_{i} \\
& \partial_{0} T \simeq 0 \\
& \partial_{0} D \simeq-2 T
\end{aligned}
$$

If $T$ and $D$ vanish initially, they vanish at all times, independently of the other three constraints. (This is true for the full evolution equations, not shown here, as well as the principal terms given in (43) and (44).) From now on we restrict attention to the subspace of solutions where these algebraic constraints vanish. A discrete version of this restriction is implemented in the "BSSN" code of most groups by projecting to solutions of $T=D=0$ at each time step. It is not clear how exactly this relates to the continuum restriction [5]. We shall call the restricted continuum system BSSN-C (C is for constrained). Note that although NOR has 15 variables and BSSN-C 17, two of those are algebraically redundant because we consider $\tilde{A}_{i j}$ to be tracefree and $\tilde{\gamma}_{i j}$ to have unit determinant.

There is a one-to-one correspondence between the variables of BSSN-C and NOR given by (26), (28), and (29) and

$$
f_{i}=\tilde{\gamma}_{i j} \tilde{\Gamma}^{j}+(4-6 \rho) \phi_{, i} .
$$

From (26) and (28) we also have the useful relations

$$
d_{i}=\tilde{\gamma}^{j k} \tilde{\gamma}_{i j, k}+4 \phi_{, i}, \quad t_{i}=12 \phi_{, i} .
$$

The constraints $H, M_{i}$, and $G_{i}$ as defined for NOR in (7), (8), and (14) and for BSSN in (30), (38), and (39) are also equivalent in the principal part modulo $T=D=0$, and we have anticipated this by using the same notation for both. Finally, if $c=-1 / 3, d=-a / 3$, and $\rho=2 / 3$, the principal parts of the evolution equations and constraint evolution equations are also equivalent (for any $a$ and $b$ ). The values of $a$ and $b$ in NOR then correspond to those of BSSN-C, and we have anticipated this by using the same notation for both.

In the following we focus on two versions of NOR: one where $a=b=1, c=d=0$, which we shall call NOR-A, and one where $a=b=1, c=d=-1 / 3$, which we shall call NOR-B. ADM is also considered in the form of NOR with $a=b=c=d=0$. In these three cases we also assume $\sigma=0$ unless specified otherwise. Our results can be summarized in the following:

Lemma 1: For any choice of lapse and shift, there is a one-to-one algebraic correspondence between the variables of BSSN-C and of NOR-B with $\rho=2 / 3$ and, using this, between the principal parts of their evolution equations, constraints, and constraint evolutions. In particular, $B S S N-C$ is strongly/symmetric hyperbolic (in the definition of [6]) if and only if NOR-B is.

\section{E. KST with fixed gauge}

BSSN-C and NOR-B are both second-order formulations and have the same number of variables, namely, 15. By contrast, the KST formulation [7] is first order and is not evidently the reduction to first order of any secondorder system [3], while a first-order reduction of NOR has 33 variables, not 30. Nevertheless, in this subsection we show that the two formulations are equivalent in the precise sense that when only the principal part is considered, the KST formulation of the Einstein equations with densitized lapse and fixed shift [7] is an autonomous subsystem, in 30 of the 33 variables, of a reversible reduction to first order of NOR with densitized lapse and fixed shift. In the presence of an evolved lapse and shift, this equivalence must be revisited because the evolution equations for the lapse and shift could be written in a first-order form which is not a reversible reduction of their second-order form. This will be done in the following two subsections.

In this subsection, we shall assume that the lapse densitization weight $\sigma$ is a given parameter, and that the parameter $\rho$ of NOR is also given. We shall prove that the remaining parameters $(a, b, c, d)$ of NOR correspond to the parameters $(\zeta, \gamma, \chi, \eta)$ of KST as follows:

$$
\begin{gathered}
4 b=2 \chi+4 \eta-\rho(3 \chi+\eta), \\
2 c=\gamma,
\end{gathered}
$$

$$
\begin{aligned}
& 4 a b=-(1+3 \sigma) \chi+\left(\frac{1}{2}-\sigma-\frac{3}{2} \zeta\right) \eta, \\
& 4 d b=-(1+2 \gamma) \chi+\left(\frac{1}{2}+\frac{1}{2} \zeta+\gamma\right) \eta .
\end{aligned}
$$

Note that $b, \chi$, and $\eta$ only appear in the combinations $\eta / b$ and $\chi / b$.

We define the shorthand

$$
\begin{aligned}
\mathcal{K}_{i j}\left(X_{k l m}, \zeta, \gamma, \sigma\right) \equiv & -\frac{1+\sigma}{2} X_{(i|k,| j)}{ }^{k}-\frac{1}{2} X_{k i j}{ }^{k} \\
& +\frac{1+\zeta}{2} X_{(i j) k}{ }^{, k}+\frac{1-\zeta}{2} X_{k}{ }^{k}(i, j) \\
& -\frac{\gamma}{2} \gamma_{i j}\left(X_{k l}^{l, k}-X_{k}^{k, l}{ }_{, l}\right),
\end{aligned}
$$

for any $X_{k l m}$ that is symmetric in its last two indices. With the parameter identifications $(48)-(50), \mathcal{K}_{i j}$ obeys the 
identity

$$
\begin{gathered}
\mathcal{K}_{i j}\left(X_{k l m}+\frac{\chi}{2 b} \gamma_{l m} Y_{k}+\frac{\eta}{2 b} \gamma_{k(l} Y_{m)}, \zeta, \gamma, \sigma\right) \\
\simeq \mathcal{K}_{i j}\left(X_{k l m}, \zeta, 2 c, \sigma\right)+a Y_{(i, j)}+d \gamma_{i j} Y_{k}{ }^{, k}
\end{gathered}
$$

for any $X_{k l m}$ symmetric in its last two indices and $Y_{i}$. Here $\simeq$ indicates that we have neglected first derivatives of $\gamma_{i j}$. We now go from NOR to KST in three steps.

\section{First-order reduction of NOR}

Using our shorthand notation, we can write the NOR evolution equations with fixed gauge in the form

$$
\begin{gathered}
\partial_{0} \gamma_{i j} \simeq-2 K_{i j}, \\
\partial_{0} f_{i} \simeq-2 K_{i k}^{, k}+\rho K_{, i}+2 b M_{i}, \\
\partial_{0} K_{i j} \simeq \mathcal{K}_{i j}\left(\partial_{k} \gamma_{l m}, *, 2 c, \sigma\right)+a G_{(i, j)}+d \gamma_{i j} G_{k}^{, k},
\end{gathered}
$$

where the $*$ indicates that the formal parameter $\zeta$ of $\mathcal{K}$ cancels because $\gamma_{i j, k l}=\gamma_{i j, l k}$. $G_{i}$ was defined above in (14).

We reduce the NOR system to first order by introducing the auxiliary variables $e_{k i j} \doteq \gamma_{i j, k}$. The new evolution equations are

$$
\begin{gathered}
\partial_{0} K_{i j} \simeq \mathcal{K}_{i j}\left(e_{k l m}, \zeta, 2 c, \sigma\right)+a \bar{G}_{(i, j)}+d \gamma_{i j} \bar{G}_{k}{ }^{, k}, \\
\partial_{0} e_{k i j} \simeq-2 K_{i j, k},
\end{gathered}
$$

with (53) and (54) as before. $\zeta$ now arises as a reduction parameter, and we have used the shorthand $\bar{G}_{i} \equiv$ $f_{i}-e^{k}{ }_{k i}-(\rho / 2) e_{i k}{ }^{k}$ for the new definition constraint. Because no constraints have been introduced in (57), the reduction is reversible in the sense defined in [6]. This means that the reduction is strongly (symmetric) hyperbolic if and only if the original second-order system is strongly (symmetric) hyperbolic. Furthermore, if both are symmetric hyperbolic, their energies are related by identifying $e_{k i j}$ and $\gamma_{i j, k}$. (See [6] for definitions of strong and symmetric hyperbolicity of second-order systems, and the relation with their first-order counterparts.)

We now decompose $e_{k i j}$ (18 components) into $\tilde{e}_{k i j}(15$ independent components) and $e_{i}$ (3 components) as follows:

$$
\begin{gathered}
\tilde{e}_{k i j} \equiv e_{k i j}-\frac{\chi}{2 b} \gamma_{i j} e_{k}-\frac{\eta}{2 b} \gamma_{k(i} e_{j)}, \\
e_{i} \equiv e^{k}{ }_{k i}-\frac{\rho}{2} e_{i k}{ }^{k}, \\
0=\tilde{e}_{k i}^{k}-\frac{\rho}{2} \tilde{e}_{i k}{ }^{k} .
\end{gathered}
$$

Here (47) is necessary for (60) to hold. The two parts of $e_{k i j}$ evolve as

$$
\begin{gathered}
\partial_{0} \tilde{e}_{k i j} \simeq \partial_{0} e_{k i j}-\frac{\chi}{2 b} \gamma_{i j} \partial_{0} e_{k}-\frac{\eta}{2 b} \gamma_{k(i} \partial_{0} e_{j)}, \\
\partial_{0} e_{i} \simeq-2 K_{i k}{ }^{k}+\rho K_{, i} .
\end{gathered}
$$

From (52) with $X_{k i j} \rightarrow e_{k i j}$ and $Y_{i} \rightarrow-e_{i}$ we see that (56) can be rewritten as

$$
\partial_{0} K_{i j} \simeq \mathcal{K}_{i j}\left(\tilde{e}_{k l m}, \zeta, 2 c, \sigma\right)+a f_{(i, j)}+d \gamma_{i j} f_{k}{ }^{, k} .
$$

The evolution equations of first-order NOR in final form are (53), (54), and (61)-(63). When only the principal part is considered, the subsystem (54), (61), and (63) in the variables $\left(\tilde{e}_{k i j}, f_{i}, K_{i j}\right)$ is autonomous, and the variables $\left(e_{i}\right.$, $\gamma_{i j}$ ) follow passively, and so could, always in the principal part approximation, be found after the subsystem has been solved. Any estimate on the subsystem gives rises to a similar estimate for the full system. Therefore the full system is strongly hyperbolic if and only if the subsystem is. (We discuss symmetric hyperbolicity in Step 3).

\section{From first-order NOR to KST}

We now define

$$
\begin{gathered}
d_{k i j} \equiv \tilde{e}_{k i j}+\frac{\chi}{2 b} \gamma_{i j} f_{k}+\frac{\eta}{2 b} \gamma_{k(i} f_{j)}, \\
\equiv e_{k i j}+\frac{\chi}{2 b} \gamma_{i j} \bar{G}_{k}+\frac{\eta}{2 b} \gamma_{k(i} \bar{G}_{j)} .
\end{gathered}
$$

Note $d_{k i j}$ is equal to $e_{k i j}$ up to a constraint. From (54) and (61), or equivalently from (25) and (57), its evolution equation is

$$
\partial_{0} d_{k i j} \simeq-2 K_{i j, k}+\chi \gamma_{i j} M_{k}+\eta \gamma_{k(i} M_{j)} .
$$

Finally we use (52) with $X_{k i j} \rightarrow \tilde{e}_{k i j}$ and $Y_{i} \rightarrow f_{i}$ to rewrite (63) as

$$
\partial_{0} K_{i j} \simeq \mathcal{K}_{i j}\left(d_{k l m}, \zeta, \gamma, \sigma\right) .
$$

(53), (66), and (67) together form the KST evolution equations for fixed gauge [7]. (Note that $\sigma \equiv 2 \sigma_{\mathrm{KST}}$ and that in [8] the notation $D_{k i j} \equiv d_{k i j} / 2$ is used.) In this second step we have discarded the dynamical variables $e_{i}$ from the autonomous evolution system. Their place in $d_{k i j}$ is taken by the $f_{i}$.

An equivalence between BSSN and a particular case of KST was established in [9] without however clarifying the role of the algebraic constraints in BSSN, or noting the special role of the $e_{i}$.

\section{Symmetric hyperbolicity}

The conserved energy of NOR with fixed gauge in its original second-order form is

$$
\begin{aligned}
\epsilon\left(K_{i j}, \gamma_{i j, k}, f_{i}\right)= & c_{0} \epsilon_{0}+c_{1} \epsilon_{1}+c_{2}\left(d_{i}^{2}-\gamma_{i j, k} \gamma^{i k, j}\right) \\
& +c_{3}\left[G_{i}+b\left(d_{i}-t_{i}\right)\right]^{2},
\end{aligned}
$$


where $c_{0} \epsilon_{0}+c_{1} \epsilon_{1}$ is a complicated expression depending on $(\rho, a, b, c, d, \sigma)$. (The special case $c=d=0$ is given in [3]). The energy of NOR in first-order form is obtained by replacing $\gamma_{i j, k}$ with $e_{k i j}$, and setting $c_{2}=(1+\zeta) / 4$. In the first-order system $\zeta$ is a reduction parameter which then determines $c_{2}$, while in the second-order system $c_{2}$ arises as a free parameter in the energy.

The main difference between KST and first-order NOR is that the latter has the additional variable $e_{i}$. However, there is a unique choice for $c_{3}$ (a complicated expression involving $(a, b, c, d, \sigma)$ but not $\rho)$ which, together with $c_{2}=(1+\zeta) / 4$, eliminates $e_{i}$ completely from the NOR energy. (This is nontrivial as three terms $e^{i} e_{i}, e^{i} f_{i}$, and $e^{i} \tilde{e}_{i k}{ }^{k}$ must be eliminated from the energy with only the one parameter $c_{3}$ to adjust.) The resulting NOR energy becomes the KST energy (compare Appendix A of [8]) when $\tilde{e}_{k i j}$ and $f_{i}$ are replaced by $d_{k i j}$ and its partial traces according to (64) and with the parameter identifications and choice of $c_{3}$ given here. Then KST with fixed gauge is symmetric hyperbolic if and only if NOR is. We summarize our results in this subsection in:

Lemma 2: There is a reduction to first order (from now, NOR1) of the second-order NOR evolution equations (from now, NOR2) with densitized lapse and fixed shift. A subsystem of NORI (from now, NORIa) is autonomous in the principal part approximation. It is possible to relate the parameters of NORI and KST such that in the principal part approximation there is a bijection between the variables and evolution equations of NORla and KST. The evolution of the variables $e_{i}$ which are in NORl but not in NORIa is trivial in the principal part approximation. This means that NORI is strongly hyperbolic if and only if KST is. This in turn means that NOR2 is strongly hyperbolic if and only if KST is.

Furthermore, NOR2 admits an energy which, when translated into first-order form, does not contain $e_{i}$ and so is an energy for NORla. There is bijection between this and the KST energy. This means that NOR2 is symmetric hyperbolic if and only if KST is.

(Here the hyperbolicity of second-order systems is the one defined in [6].)

\section{F. KST with evolved lapse: Sarbach-Tiglio}

The Sarbach-Tiglio (ST) formulation of the Einstein equations [10] is KST with the evolved lapse

$$
\partial_{0}(\ln \alpha) \simeq-\mu_{L} K
$$

where $\mu_{L}=2 \sigma_{\text {eff }}=\alpha^{-1} \partial F / \partial K$ in the notation of [10]. We now show that the ST formulation is (an autonomous subsystem of) a reversible reduction to first order of NOR with this lapse. We start again from NOR and work towards ST.

The live lapse NOR evolution equations are (53) and (54) together with

$$
\begin{aligned}
\partial_{0} K_{i j} \simeq & -(\ln \alpha)_{, i j}+\mathcal{K}\left(g_{i j, k}, *, 2 c, 0\right)+a G_{(i, j)} \\
& +d \gamma_{i j} G_{k}{ }^{, k} .
\end{aligned}
$$

We introduce the auxiliary variable

$$
a_{i} \doteq(\ln \alpha)_{, i}
$$

and evolve it as

$$
\partial_{0} a_{i} \simeq-\mu_{L} K_{, i}
$$

so that its introduction is reversible. In analogy with (65) we now define

$$
A_{i} \equiv a_{i}+\frac{\xi}{2 b} G_{i}
$$

which obeys

$$
\partial_{0} A_{i} \simeq-\mu_{L} K_{, i}+\xi M_{i}
$$

with $\xi$ a new parameter. We complete the reduction to first order as in the fixed gauge case by introducing $e_{k i j}$, then replacing its components $e_{i}$ by $f_{i}$ to obtain $d_{k i j}$. This transforms (70) into

$$
\partial_{0} K_{i j} \simeq-A_{(i, j)}+\mathcal{K}\left(d_{k i j}, \zeta, \gamma, 0\right) .
$$

(53), (66), (74), and (75) form the ST evolution equations, where the NOR and KST parameters are related by (47), (48), and (50) with (49) replaced by

$$
4 a b=-\chi+\left(\frac{1}{2}-\frac{3}{2} \zeta\right) \eta-2 \xi .
$$

Compared to (49), we have set $\sigma=0$, and the additional term $-2 \xi$ appears on the right-hand side because of the appearance of $(2 \xi / b) G_{(i, j)}$ in $A_{(i, j)}$. We have shown that ST is equivalent to a first-order reduction of NOR with the lapse evolution (69). The characteristic speeds squared of [10] simplify in our notation to $\lambda_{1}=\mu_{L}, \lambda_{2}=1+4(c-$ $b d)$ and $\lambda_{3}=a b$.

Now hold $\mu_{L}$ fixed and set $\sigma=0$. Then NOR has 5 parameters $(a, b, c, d, \rho)$ and KST has 5 parameters $(\gamma, \zeta$, $\chi, \eta, \xi)$, but there are only 4 algebraic relations between them, namely $2 c=\gamma$, and (for generic values of $\rho$ ) three linear relations between $(b, a b, b d)$ and $(\chi, \eta, \xi)$. One can consider $\rho$ in NOR and $\zeta$ in KST as arbitrary in their system with no counterpart in the other evolution system.

ST find a 3-parameter family of strongly hyperbolic formulations whose NOR equivalent is $a b=1, d=a c$. Their 2-parameter family is the special case $c=-1 / 4$. The 3 free parameters can be taken to be $(\gamma, \zeta, \eta)$ in KST and $(\rho, a, c)$ in NOR, with only 2 algebraic relations between these sets. ST also find a 2-parameter family of symmetric hyperbolic formulations, which corresponds to NOR with $c=-1 / 3, d=-7 / 30$. The free parameters can be taken to be $(a, b, \rho)$ on the NOR side but on the KST side there are only two, for example, $(\eta, \xi)$, which are determined by $(a, b)$ alone. 
Note finally that the equivalence between NOR and KST would hold for any gauge that specifies the first time derivative of the lapse, with fixed shift.

Lemma 3: Lemma 2 holds also for NOR and KST with a Bona-Massó type evolved lapse and fixed shift.

\section{G. KST with evolved lapse and shift: Lindblom-Scheel}

The formulation of Lindblom and Scheel (LS) [8] is again based on KST, but with an evolved shift as well as lapse. The gauge conditions are (111) and (112) below, but the following comments apply to any gauge where the first time derivatives of the lapse and shift are specified. Besides the auxiliary variables $d_{k i j}$ and $A_{i}$ already present in ST (called $2 D_{k i j}$ and $T_{i}$ in LS), the auxiliary variable $M_{k}{ }^{i} \doteq$ $\alpha^{-1} \beta_{, k}^{i}$ is introduced, and all possible constraints with the right number of indices and level of derivatives are added to the right-hand side of its evolution equation. In our notation, these are

$$
\partial_{0} M_{k}{ }^{i}=\ldots+\tilde{\psi}_{4} G_{k}{ }^{i}+\tilde{\psi}_{5} G^{i}{ }_{, k}+\tilde{\psi}_{6} \delta_{k}{ }^{i} G_{l}{ }^{l}+\tilde{\psi}_{7} \delta_{k}{ }^{i} H,
$$

plus auxiliary constraints. (Our constants $\tilde{\psi}_{4 . \ldots 7}$ are linear combinations of $\psi_{4 \ldots 7}$ of LS. The derivatives of $G_{i}$ are added not in this explicit form but implicitly with the definition constraints of $d_{k i j}$ and $A_{i}$. We think of $G_{i}$ as the relevant part of those constraints, and the definition constraints of $a_{i}$ and $e_{k i j}$ as the part that is irrelevant because its addition does not change the reversibility of the first-order reduction.) Because there are no constraints whose time derivatives are equal to these constraint terms, we cannot use a trick analogous to (73) and (74) to recreate the effect of these constraint additions in the first-order reduction within fully second-order NOR. The smallest system fully equivalent to LS that could be constructed would be a hybrid between second-order NOR and LS comprising the NOR variables $\left(\gamma_{i j}, K_{i j}, f_{i}\right)$ and gauge variables $\left(\alpha, \beta^{i}\right)$ but also $M^{i}{ }_{k}$. We have not analyzed this further.

Conversely, the introduction of $M^{i}{ }_{k}$ remains reversible if we set $\tilde{\psi}_{4 . .7}=0$. In the notation of LS, this corresponds to the conditions

$$
\begin{gathered}
16 \chi \xi \psi_{3}+\left(8 \chi^{2}+8 \chi \eta+3 \eta^{2}\right) \psi_{4}=0 \\
\eta \psi_{4}-4 \chi \psi_{5}=0 \\
\psi_{5}-\psi_{6}=0 \\
\psi_{7}=0
\end{gathered}
$$

on $\psi_{4 \ldots . .7}$. With these restrictions, LS is a reversible firstorder reduction of NOR with the same gauge. The identification between NOR and LS parameters is then given by (47), (48), (50), and (76) and $\psi_{1}=2 \xi$. The remaining free parameters $\left(\psi_{2}, \psi_{3}, \psi_{8}, \psi_{9}, \psi_{10}\right)$ of LS are reduction parameters connected to the introduction of auxiliary variables. They have no direct counterpart in the NOR evolution equations but can be identified with free parameters in the NOR energy.

Lemma 4: Lemma 2 holds also for NOR with a Lindblom-Scheel-type evolved lapse and shift and the subset of LS formulations characterized by the parameter restrictions $(78)-(81)$.

\section{ANALYSIS OF LIVE GAUGE CONDITIONS}

\section{A. Symmetry-seeking coordinate choices}

In the $3+1$ approach the gauge freedom of general relativity is fixed by specifying the lapse $\alpha$ and shift $\beta$ in terms of the 3-metric $\gamma_{i j}$ and the extrinsic curvature $K_{i j}$, and possibly given functions of $\left(t, x^{i}\right)$, through algebraic or differential equations.

A unifying viewpoint for the classification of gauge conditions was given in [11]. A good gauge choice should have the property that if the spacetime is stationary the vector field $(\partial / \partial t)^{a}=\alpha n^{a}+\beta^{a}$ becomes equal to the Killing vector, whatever the initial slice and the spatial coordinates on it are. We do not require that $(\partial / \partial t)^{a}$ be timelike but only that $\alpha=-n_{a}(\partial / \partial t)^{a}>0$, where $n_{a}$ is the unit normal on the surfaces of constant $t$. This is assumed in the following. In such coordinates, $\gamma_{i j}$ and $K_{i j}$, as well as $\alpha$ and $\beta^{i}$ become independent of $t$. Such conditions can be formulated in terms of the vanishing of $3+1$ time derivatives. Modifying the terminology of [11] we shall use the term "freezing conditions" for gauge conditions in which the metric is immediately timeindependent in the presence of a Killing vector, and restrict the term "symmetry-seeking" for gauge conditions in which the metric becomes time-independent asymptotically.

\section{B. Elliptic freezing conditions}

If the dynamical variables are frozen in the presence of a Killing vector, so are the lapse and shift. Freezing conditions for the lapse and shift can therefore not contain their time derivatives. An example is the $K$-freezing lapse

$$
\dot{K}=0 .
$$

For given $\beta^{i}, \gamma_{i j}$, and $K_{i j}$, this is an elliptic equation for $\alpha$, whose principal part is the Laplace operator associated with $\gamma_{i j}$. It is clear from the way we have written it that this is a freezing coordinate condition. Irrespective of the evolution system, we always use the simplest form

$$
\dot{K}=\beta^{i} K_{, i}-\Delta \alpha+\alpha K_{i j} K^{i j}
$$

in any gauge condition. The principal part of this can be written as

$$
\partial_{0} K \simeq-\partial_{i} \partial^{i}(\ln \alpha)
$$


If $K=0$ in the original slice (and therefore on all slices), the $K$-freezing lapse is called maximal slicing. Maximal slicing combined with minimal strain shift

$$
D^{i} \dot{\gamma}_{i j}=0
$$

or minimal distortion shift

$$
D^{i} \dot{\tilde{\gamma}}_{i j}=0
$$

were first suggested for use in numerical relativity in [12].

If, neglecting lower-order terms, we pull the time derivative out of the divergence, we can integrate these last two gauge conditions over $t$. In the context of the BSSN formulation, the gauge condition $\dot{\tilde{\Gamma}}^{i}=0$ has been considered under the name $\Gamma$-freezing shift. We consider a generalization in the form of the 2-parameter family of shift conditions

$$
\dot{\bar{f}}^{i}=0
$$

where

$$
\begin{gathered}
\bar{f}_{i} \equiv d_{i}-\frac{\bar{\rho}}{2} t_{i}+s G_{i} \\
=s f_{i}+(1-s) d_{i}+\frac{s \rho-\bar{\rho}}{2} t_{i}, \\
=s \tilde{\gamma}_{i j} \tilde{\Gamma}^{j}+(1-s) d_{i}+\left(\frac{s}{3}-\frac{\bar{\rho}}{2}\right) t_{i},
\end{gathered}
$$

where $\bar{\rho}$ and $s$ are constant parameters. Essentially, $\bar{f}^{i}$ is the divergence of the 3 -metric. The price we pay for pulling the time derivative outside the divergence is that while $\dot{\gamma}_{i j}$ transforms as a tensor under spatial coordinate transformations, $\bar{f}^{i}$ does not. (However, $\bar{f}^{i}=0$ and similar conditions (Dirac gauge) can be formally made covariant by writing $f^{i}$ as the covariant divergence of $\gamma_{i j}$ with respect to a flat background metric [13]). $\bar{\rho}$ parametrizes a conformal weight, and $s=1$ gives us the option to express the gauge condition in terms of the NOR variable $f^{i}$, while with $s=$ 0 the gauge condition is expressed in terms of the 3-metric. With $s=1, \rho=\bar{\rho}=2 / 3$ we have $\bar{f}^{i}=\gamma^{-1 / 3} \tilde{\Gamma}^{i}$.

We shall loosely call all these gauge conditions $\Gamma$-freezing. They are elliptic in $\beta^{i}$ for $\bar{\rho}<2$, and by construction are freezing. The principal part of $\dot{\bar{f}}^{i}=0$ can be read off from

$$
\begin{aligned}
\partial_{0} \bar{f}_{i} \simeq & \alpha^{-1} \gamma_{i k} \beta^{k}{ }_{, j}{ }^{j}+(1-\bar{\rho}) \alpha^{-1} \beta^{j}{ }_{, j i}+2(s b-1) K_{i j}{ }^{j} \\
& +(\bar{\rho}-2 s b) K_{, i} .
\end{aligned}
$$

The principal part in $\beta^{i}$ coincides with minimal strain shift and minimal distortion shift for $\bar{\rho}=0$ and $\bar{\rho}=2 / 3$, respectively.

\section{Parabolic drivers}

Coordinate drivers turn elliptic equations for the lapse or shift into heat equations or wave equations by adding one or two time derivatives. Their motivation is that in a spacetime with a Killing vector, and with suitable boundary conditions, we expect that the solution of the resulting heat or wave equation will tend towards a (timeindependent) solution of the original elliptic freezing condition.

Parabolic drivers for the lapse and shift were, to our knowledge, first suggested in [14]. The basic (explicit) parabolic " $K$-driver" is

$$
(\ln \alpha)^{\cdot}=-\nu_{L} \dot{K}
$$

for some constant $\nu_{L}>0$. The choice of powers of $\alpha$ here and in the following is determined by the requirement that the gauge choice be invariant under the rescaling of $t$ by a constant factor. The equation is also invariant under arbitrary changes of the spatial coordinates.

The elliptic equation $\dot{K}=0$ has been turned into a heat equation for $\alpha$. If all variables other than $\alpha$ were timeindependent, the solution of the parabolic equation would tend to a solution of the elliptic equation $\dot{K}=0$, but in reality all variables evolve together. Whether the solution actually tends towards a freezing condition will therefore depend on the physical problem. However, we can see that the driver is at least compatible with being symmetryseeking in the sense that the evolution of $\alpha$ stops if and only if the freezing condition $\dot{K}=0$ is obeyed.

Integrating explicitly over $t$, we obtain the basic implicit parabolic $K$-driver

$$
\ln \alpha=-\nu_{L} K+\phi(x) .
$$

As $K$ is a dynamical variable, this is now an algebraic gauge condition rather than a heat equation, and so the implicit and explicit forms are not equivalent as systems of partial differential equations (PDEs). However, if the constraints are obeyed the two forms will generate the same gauge. In particular, $\phi(x)$ allows us to still set gauge initial data for $\alpha$ independently of the value of $K$ given by the geometric initial data, as

$$
\phi(x)=\left(\nu_{L} K+\ln \alpha\right)_{0} .
$$

(In the remainder of this section the suffix 0 denotes the initial value of a quantity.) On the other hand, if the parabolic driver succeeds in driving $K$ to a timeindependent value, $\phi(x)$ also characterizes a condition on the asymptotic values of $K$ and $\alpha$, namely

$$
\phi(x)=\left(\nu_{L} K+\ln \alpha\right)_{\infty},
$$

where the suffix $\infty$ denotes the asymptotic value. Such a condition is to be expected: while elliptic conditions freeze $K$ and $\bar{f}^{i}$ at whatever value they have in the initial data, a driver condition freezes $K$ and $\bar{f}^{i}$ only asymptotically and at values which depend not only on the geometric initial 
data $K_{i j}$ and $\gamma_{i j}$, but also on the initial values of $\alpha$ and $\beta^{i}$ (and $\dot{\alpha}$ and $\dot{\beta}^{i}$ in the case of a hyperbolic driver, see below).

The situation for the $\Gamma$-freezing shift conditions is very similar to the $K$-freezing lapse condition. The basic parabolic " $\Gamma$-driver" is

$$
\dot{\beta}^{i}=\nu_{S}\left(\alpha \bar{f}^{i}\right)^{.}
$$

for some constant $\nu_{S}$. The left-hand side is a vector and the right-hand side is not (which means that the time evolution does not commute with generic spatial coordinate transformations), but the equation transforms correctly under rescaling the spatial coordinates by a constant factor and under rotations. The factor of $\alpha$ makes the equation invariant under rescaling $t$ by a constant factor. For $\bar{\rho}=0$ this is a heat equation for $\beta^{i}$, and for $\bar{\rho}<2$ it is parabolic. Again, this condition is compatible with being symmetry-seeking. Similarly, an implicit parabolic shift condition is

$$
\beta^{i}=\nu_{S} \alpha \bar{f}^{i}-\phi^{i}(x) .
$$

What would happen if we replaced $\partial_{t}$ with $\partial_{0}$ ? The straightforward replacement,

$$
\partial_{0}(\ln \alpha)=-\nu_{L} \partial_{0} K
$$

can be integrated using the method of lines:

$$
\ln \alpha+\nu_{L} K=\phi[\tilde{x}(x, t)],
$$

where

$$
\dot{\tilde{x}}^{i}=\beta^{j} \tilde{x}_{, j}^{i}, \quad \tilde{x}^{i}(x, 0)=x^{i},
$$

and $\phi(x)$ is given by (94). Clearly this couples the lapse and shift conditions through $\tilde{x}^{i}$, leading to a gauge condition which is different from the original one. However, if we write

$$
\partial_{0}(\ln \alpha)=-\nu_{L} \partial_{0} K+\alpha^{-1} \beta^{i} \partial_{i} \phi(x),
$$

this can be rewritten as the $\partial_{0}$ derivative of (93). If we again define $\phi(x)$ from (94), we have (93) identically. Taking a $\partial_{t}$ derivative, we obtain (92). Therefore (92) and (101) give rise to the same gauge (when all Einstein equations are obeyed). In particular we have (95). However, the $\partial_{0}$ and $\partial_{t}$ implementations have different principal parts, and so their well-posedness is not obviously equivalent. The use of $\partial_{0}$ in the gauge conditions simplifies the well-posedness analysis, but $\partial_{0} \beta^{i}$ is similar to the nonlinear term in Burger's equation, and may develop shocks [15]. Contrary to the claim in [8], this problem cannot be fixed simply by introducing an auxiliary variable for the first derivatives of the shift, as the reduction admits the same blow-up solutions.

\section{Hyperbolic drivers}

Hyperbolic drivers were discussed in [16]. The basic explicit hyperbolic $K$-driver is

$$
\left(\alpha^{-1}(\ln \alpha)^{\cdot}\right)^{\cdot}+2 \kappa_{L}(\ln \alpha)^{\cdot}=-\mu_{L} \dot{K},
$$

for some $\mu_{L}>0$ and $\kappa_{L}>0$. This equation has the same transformation properties as the parabolic lapse driver. The motivation is that the elliptic equation $\dot{K}=0$ will be solved by turning it into a wave equation with a friction term for $\alpha$. Once again, this would be true if all variables other than $\alpha$ were time-independent but it is not clear if this gauge condition is symmetry-seeking in general when it is applied to a situation where all dynamical variables evolve.

As we have chosen the outermost derivative to be $\partial_{t}$, we can again integrate in $t$ to obtain the basic implicit hyperbolic $K$-driver

$$
\dot{\alpha}=-\alpha^{2} \mu_{L} K-2 \kappa_{L} \alpha^{2} \ln \alpha+\alpha^{2} \phi(x),
$$

which is again equivalent as a gauge condition to the explicit driver as long as the constraints are obeyed. $\phi(x)$ can be used to set initial data for $\dot{\alpha}$ independently of $\alpha$ and $K$, but if the spacetime admits a Killing vector and the driver is indeed symmetry-seeking then it is also related to the asymptotic values of $\alpha$ and $K$ :

$$
\phi(x)=\left(\mu_{L} K+2 \kappa_{L} \ln \alpha\right)_{\infty} .
$$

Another damping mechanism (suggested by Shibata [17] for the shift driver) for an implicit hyperbolic driver is

$$
\dot{\alpha}=-\mu_{L} \alpha^{2} K-2 \nu_{L} \alpha \dot{K}+\alpha^{2} \phi(x) .
$$

By linearizing in $\alpha$, holding all other variables fixed and carrying out a Fourier analysis, one sees that all modes are damped. However, this linear equation for $\alpha$ (with all other variables held fixed) is neither hyperbolic nor parabolic. Shibata chooses $\eta \propto \Delta x$, so that in the continuum limit the implicit hyperbolic driver is recovered. One advantage of this damping mechanism is that now $\phi(x)=\mu_{L} K_{\infty}$, giving better control of the final gauge.

Again, a similar analysis applies to the $\Gamma$-freezing shift conditions. The basic explicit hyperbolic $\Gamma$-driver (with damping) is

$$
\left(\alpha^{-1} \dot{\beta}^{i}\right)^{\cdot}+2 \kappa_{S} \dot{\beta}^{i}=\left(\mu_{S} \alpha \bar{f}^{i}\right)^{.} .
$$

(This equation has the same transformation properties as the parabolic shift driver.) For $\bar{\rho}=0$ this is a wave equation for $\beta^{i}$. The equivalent implicit hyperbolic $\Gamma$-driver is

$$
\dot{\beta}^{i}=\mu_{S} \alpha^{2} \bar{f}^{i}-2 \kappa_{S} \alpha \beta^{i}-\alpha \phi^{i}(x),
$$

and the alternative damping mechanism is

$$
\dot{\beta}^{i}=\mu_{S} \alpha^{2} \bar{f}^{i}+2 \nu_{S} \alpha \dot{\bar{f}}^{i}-\alpha \phi^{i}(x),
$$

the latter obeying $\phi^{i}(x)=\mu_{S} \alpha \bar{f}_{\infty}^{i}$.

Finally we note that the explicit parabolic or hyperbolic drivers differ from the explicit ones in that in the expressions for $\dot{K}$ or $\dot{\bar{f}}^{i}$ that appear on their right-hand side one can add suitable constraints in order to change the principal part of the equations, while this is not possible in the implicit case. 
We can strip the outer time derivative off the explicit hyperbolic drivers to obtain the implicit hyperbolic drivers even if that outer time derivative is $\partial_{0}$ with the same trick of adding a source term we used above in (101). This affects the nature and well-posedness of the system, but leaves the gauge choice unaffected. This is not true if we replace the remaining (inner) time derivative $\partial_{t}$ with $\partial_{0}$ on the left-hand side of the implicit hyperbolic drivers. For example, with

$$
\partial_{0}(\ln \alpha)=-\mu_{L} K-2 \kappa_{L} \ln \alpha+\phi(x),
$$

(104) is replaced by

$$
\phi=\left(\mu_{L} K+2 \kappa_{L} \ln \alpha+\alpha^{-2} \beta^{i} \alpha_{, i}\right)_{\infty},
$$

which couples the asymptotic lapse and shift.

\section{E. Boundary conditions}

Elliptic gauge conditions guarantee that 4 dynamical variables are frozen in any spacetime, but $\gamma_{i j}, K_{i j}, \alpha$, and $\beta^{i}$ are all frozen in a stationary spacetime only if the boundary conditions are compatible with the Killing vector. Finding boundary conditions which are at the same time compatible with the symmetry-seeking gauge, the constraints, and give rise to a well-posed initial-boundary value problem is an important problem that has not yet been studied in any depth. The parabolic and hyperbolic drivers require the same type of boundary conditions as the elliptic freezing conditions, namely, Dirichlet, Neumann, or mixed boundary conditions for the lapse and shift. These cannot be completely homogeneous, as they must fix an overall common factor in the lapse and shift (corresponding to the freedom to rescale $t$ ). We shall not otherwise investigate this issue here.

Finally, we note that when the gauge evolution uses $\partial_{0}$ and a symmetric hyperbolic evolution system is obtained, one can arrange the characteristic speeds so that singularity excision surfaces inside black holes become purely outflow, and no boundary conditions are required. When the hyperbolic gauge evolution uses $\partial_{t}$, or when the gauge condition is elliptic or parabolic, boundary conditions for the gauge are required at excision boundaries. This may be desirable or undesirable. From the fact that the Einstein equations are well-posed in harmonic gauge, where excision surfaces are purely outflow, it is clear that no physical information can leave the black hole even if boundary conditions are imposed inside.

\section{HYPERBOLICITY WITH IMPLICIT HYPERBOLIC LAPSE AND SHIFT DRIVERS}

\section{A. Generalized Lindblom-Scheel gauge}

In the following we investigate the strong and symmetric hyperbolicity of ADM, NOR-A, and NOR-B coupled to a family of implicit hyperbolic driver gauge conditions. These conditions are

$$
\begin{gathered}
\left(\partial_{0}+\zeta_{L} \partial_{\bar{\beta}}\right)(\ln \alpha) \simeq-\mu_{L} K+\epsilon_{L} \alpha^{-1} \beta^{i}{ }_{, i}, \\
\left(\partial_{0}+\zeta_{S} \partial_{\bar{\beta}}\right) \beta^{i} \simeq \mu_{S} \alpha \bar{f}^{i}+\epsilon_{S} \alpha(\ln \alpha)^{, i},
\end{gathered}
$$

where we have not written the friction damping terms and the integration constants $\phi, \phi^{i}$ because they are nonprincipal. We have introduced the shorthands $\bar{\beta}^{i} \equiv \alpha^{-1} \beta^{i}$ and $\partial_{\bar{\beta}} \equiv \bar{\beta}^{i} \partial_{i}$.

The free parameters in the principal part of the gauge conditions are $\bar{\rho}, \zeta_{L}, \zeta_{S}, \mu_{L}, \mu_{S}, \epsilon_{L}$, and $\epsilon_{S}$. With $\zeta_{L}=$ $\zeta_{S}=0$ these are the conditions considered by Lindblom and Scheel [8] and the parameters $\mu_{L}, \mu_{S}, \epsilon_{L}$, and $\epsilon_{S}$ have the same definition. The parameter $\bar{\rho}$ is related to the parameter $\lambda$ of [8] by $\bar{\rho}=1+\lambda . \zeta_{L}=\zeta_{S}=0$ simplifies the analysis of hyperbolicity, while $\zeta_{L}=\zeta_{S}=1$ corresponds to the hyperbolic drivers we have discussed above and gives rise to simpler asymptotic gauge conditions. Harmonic gauge is the special case $\zeta_{L}=\zeta_{S}=0, \mu_{L}=$ $\mu_{S}=1, \epsilon_{L}=0, \epsilon_{S}=-1, \bar{\rho}=1$. We shall refer to (111) and (112) in generality as LS gauge.

The parameter $s$ appears implicitly in the gauge conditions, but its value does not affect the gauge as long as the constraints are obeyed, and it should therefore be considered as a parameter of the formulation. Both NOR-A and NOR-B have the free parameter $\rho$, but in the following examples neither the characteristic speeds nor the wellposedness depend on the value of $\rho$.

\section{B. Using $\partial_{0}$}

We now follow [8] and investigate (111) and (112) with $\zeta_{L}=\zeta_{S}=0$, thus using $\partial_{0}$ as the time derivative. In the principal part, frozen coefficient approximation the equations decouple into three blocks, which we consider in turn. The statements below hold for ADM, NOR-A, and NOR$\mathrm{B}$, unless further qualified.

The transverse traceless symmetric tensor block of the principal part is always diagonalizable, with $\lambda= \pm 1$ (twice). The transverse vector block has eigenvalues $\lambda=$ \pm 1 (for NOR) or 0 (twice, for ADM), plus $\lambda= \pm \sqrt{\mu_{S}}$. It is diagonalizable for $\mu_{S}>0$ in the ADM case, and for $\mu_{S}>0$ with either $\mu_{S} \neq 1$ or $s=1$ in the NOR-A and NOR-B cases.

The scalar block always has $\lambda=0, \pm 1, \pm v_{ \pm}$, where

$$
\begin{gathered}
v_{ \pm}^{2} \equiv A \pm B, \\
2 A \equiv \mu_{L}+(2-\bar{\rho}) \mu_{S}+\epsilon_{S} \epsilon_{L}, \\
B^{2}-A^{2} \equiv(2-\bar{\rho})\left(\epsilon_{L}-\mu_{L}\right) \mu_{S} .
\end{gathered}
$$

$v_{ \pm}$depend only on the gauge choice, not the parameters $\rho$, $s$ of the formulation. Defining $B$ to be positive, they are real if and only if $0 \leq B \leq A$. At generic points in the interior of this region the scalar sector is diagonalizable because the four gauge speeds are different. On the lines $A=B$, $B=0$ and $A-B=1, A+B=1$ some of the character- 
istic speeds are repeated, and generically these marginal cases are not diagonalizable. In particular, $A=B$ (i.e. $v_{-}=0$ ) happens for $\bar{\rho}=2$ or $\mu_{S}=0$ or $\epsilon_{L}=\mu_{L}$ and then the system is never diagonalizable. There are further parameter choices where some of the marginal cases become diagonalizable, but we do not analyze them here. We just mention that under the assumption $A \neq B$ and using the fact that for ADM, NOR-A, and NOR-B we have $c-$ $b d=0$, diagonalizability of the whole $7 \times 7$ scalar sector with eigenvalues $\left(0, \pm 1, \pm v_{-}, \pm v_{+}\right)$is equivalent to diagonalizability of the matrix

$$
\left(\begin{array}{ccc}
\epsilon_{L} \epsilon_{S}+(2-\bar{\rho}) \mu_{S} & -\epsilon_{L} & 0 \\
-\mu_{L} \epsilon_{S}-(2-\bar{\rho}) \mu_{S} & \mu_{L} & 0 \\
-\mu_{L} \epsilon_{S}-(2 b s-\bar{\rho}) \mu_{S} & 1-2 a b+\mu_{L} & 1
\end{array}\right)
$$

with eigenvalues $\left(1, v_{-}^{2}, v_{+}^{2}\right)$.

The results simplify if we partially decouple the lapse and shift conditions by setting $\epsilon_{L}=0$. Then $v_{ \pm}$are simply $\sqrt{\mu_{L}}$ and $\sqrt{(2-\bar{\rho}) \mu_{S}}$ and the matrix (116) becomes triangular, which allows a simple discussion of its diagonalizability: as we said, we always need $\mu_{L}>0, \mu_{S}>0, \bar{\rho}<2$ to avoid a zero eigenvalue $v_{-}^{2}$. If the three eigenvalues are different then diagonalizability is guaranteed for both $\mathrm{ADM}$ and NOR. If $\mu_{L}=1$ then we need $a b=1$, which is only possible for NOR; if $(2-\bar{\rho}) \mu_{S}=\mu_{L}$ we need $\epsilon_{S}=-1$; finally if $(2-\bar{\rho}) \mu_{S}=1$ we need $\left(\mu_{L} \epsilon_{S}+1\right) \times$ $(1-a b)=\mu_{S}(b s-1)\left(\mu_{L}-1\right)$, which requires $b s=1$ for NOR-A/B. It is possible to make all scalar speeds equal to \pm 1 using the intersection of those three cases: $\epsilon_{L}=0$, $\epsilon_{S}=-1, a b=b s=\mu_{L}=1, c=b d$, and $(2-\bar{\rho}) \mu_{S}=$ 1 , while $s, d, \rho, \bar{\rho}, \sigma$ still are free parameters. We can set all speeds (including vector speeds) equal to zero or one by further setting $\mu_{S}=s=1$, which then requires $\bar{\rho}=1$. The result is the implicit form of harmonic gauge in NOR with $a=b=1$ and $c=d$, while $d, \rho, \sigma$ are still free parameters. For example, setting $d=-1 / 3, \rho=2 / 3$, and $\sigma=0$ we obtain a system equivalent to BSSN-C with implicit harmonic gauge. (Note that although the gauge is a harmonic gauge, this system is not equivalent to the usual harmonic evolution system.)

In all other cases we cannot make all speeds equal to zero or one. This is not necessarily bad: one may want constraint-violating modes to have speeds less than 1 so that they do not pile up on the black-hole horizon [8], and one may want gauge modes to have speeds larger than 1 so that one can impose boundary conditions on them at the black-hole excision boundary (inside the horizon) in order to control the gauge better [18]. We have not investigated these issues here. A simple example is

$$
\bar{\rho}=2 / 3, \quad \mu_{L}=\mu_{S}=\frac{1}{4}, \quad \epsilon_{L}=\epsilon_{S}=0,
$$

which has speeds $\lambda=(0, \pm 1, \pm 1 / 2, \pm 1 / \sqrt{3}) \leq 1$, independently of $\rho$ and $s$.
Finally we note that the lapse driver alone, with fixed shift, is strongly hyperbolic with NOR-A/B for $\mu_{L}>0$, but not with ADM.

\section{Using $\partial_{t}$}

We now set $\zeta_{L}=\zeta_{S}=1$, which is equivalent to replacing $\partial_{0}$ by $\alpha^{-1} \partial_{t}$ as the time derivative on the left-hand side of (111) and (112). As before all speeds $\lambda$ are with respect to normal observers and in units of the speed of light.

The characteristic speeds in the vector sector are $\lambda=$ \pm 1 (for NOR) or 0 (twice, for ADM), plus

$$
\lambda=-\frac{\bar{\beta}_{n}}{2} \pm \sqrt{\left(\frac{\bar{\beta}_{n}}{2}\right)^{2}+\mu_{S}}
$$

With ADM, the vector sector is diagonalizable for $\mu_{S}>0$, for any $\bar{\beta}_{n}$. With NOR, $\mu_{S}>0$ is also necessary, but not sufficient: two speeds which are generically distinct coincide for $\mu_{S}-1= \pm \bar{\beta}_{n}$, and we need to impose $\mu_{S}-1 \neq$ $\pm \bar{\beta}_{n}$ to maintain diagonalizability.

The fact that this condition depends on $\bar{\beta}_{n}=\bar{\beta}^{i} \omega_{i} /|\omega|$ raises a serious problem: As long as $|\bar{\beta}| \equiv \sqrt{\bar{\beta}^{i}} \bar{\beta}_{i}>\mid \mu_{S}-$ 1|, this inequality will be violated for all $\omega_{i}$ that lie on a cone in wave number space. In particular, it is then violated for arbitrarily large $|\omega|$. This means that for sufficiently large shift, the system is only weakly hyperbolic. The inequality required for strong hyperbolicity is therefore

$$
|\bar{\beta}|<\left|\mu_{S}-1\right| \text {. }
$$

Numerical evolutions should be unstable when it is violated. However for $s=1$ (119) is not required.

The characteristic speeds in the scalar sector are $\lambda=0$, \pm 1 and the four roots of the quartic equation

$$
\begin{aligned}
\lambda^{4}+\left(\zeta_{L}+\zeta_{S}\right) \bar{\beta}_{n} \lambda^{3}+ & {\left[\zeta_{L} \zeta_{S} \bar{\beta}_{n}^{2}-\epsilon_{L} \epsilon_{S}+(\bar{\rho}-2) \mu_{S}-\mu_{L}\right] \lambda^{2} } \\
+[(\bar{\rho}-2) & \left.\zeta_{L} \mu_{S}-\zeta_{S} \mu_{L}\right] \bar{\beta}_{n} \lambda \\
& +\left(\epsilon_{L}-\mu_{L}\right) \mu_{S}(\bar{\rho}-2)=0
\end{aligned}
$$

For $\zeta_{L}=\zeta_{S}=0$ this is a biquadratic equation and its solution is given in (113)-(115). For $\zeta_{L}=\zeta_{S}=1$ the roots are more complicated. If we restrict to $\epsilon_{L}=0$, we find that they are

$$
\begin{aligned}
\lambda= & -\frac{\bar{\beta}_{n}}{2} \pm \sqrt{\left(\frac{\bar{\beta}_{n}}{2}\right)^{2}+\mu_{L}}-\frac{\bar{\beta}_{n}}{2} \\
& \pm \sqrt{\left(\frac{\bar{\beta}_{n}}{2}\right)^{2}+(2-\bar{\rho}) \mu_{S} .}
\end{aligned}
$$

Diagonalizability of the scalar sector of ADM and NOR-A/ $B$ with LS gauge is guaranteed in the generic case where (with $\epsilon_{L}=0$ )

$$
(2-\bar{\rho}) \mu_{S}-\mu_{L} \neq 0,
$$

$$
\left|\mu_{L}-1\right|>|\bar{\beta}|,
$$




$$
\left|(2-\bar{\rho}) \mu_{S}-1\right|>|\bar{\beta}| .
$$

(Note that the equivalent conditions for the $\partial_{0}$ case are obtained by setting $\bar{\beta}^{i}$ to zero here.) However, with $a b=1$ condition (123) is not necessary, and (124) is not necessary for NOR with $s=1$.

In conclusion NOR-A/B with LS gauge can be made strongly hyperbolic with $s=1$ for all values of $\beta^{i}$, at least for $\epsilon_{L}=0$.

Finally we note that the lapse driver alone, with fixed shift, is strongly hyperbolic for NOR-B, but not for ADM (the vector sector fails to be diagonalizable) or NOR-A (the scalar sector fails to be diagonalizable. The same result was found in the ST system [10], which is equivalent to NOR with the lapse driver and fixed shift.

\section{Symmetric hyperbolicity \\ 1. NOR with live lapse and fixed shift}

The most general conserved energy has the form

$$
\epsilon=\sum_{i=0}^{5} c_{i} \epsilon_{i}
$$

with

$$
\begin{gathered}
\epsilon_{0}=K_{i j} K^{i j}+\frac{1}{4} \gamma_{i j, k}^{2}+d^{i} Y_{i}-\left(a d^{i}+d t^{i}\right) X_{i} \\
+\frac{a b-1}{2} d^{i} d_{i}+\frac{c-b d}{2} t^{i} t_{i}+\frac{1-2 a b+\mu_{L}}{2} d^{i} t_{i} \\
\epsilon_{1}=K^{2}+t^{i}\left[Y_{i}-(a+3 d) X_{i}\right. \\
\left.+\frac{1}{4}\left(2-2 a b+6 c+6 b d+\mu_{L}\right) t_{i}\right] \\
\epsilon_{2}=\gamma^{i j}\left(d_{i} d_{j}-\gamma^{k n} \gamma^{l m} \gamma_{i k, l} \gamma_{j m, n}\right) \\
\epsilon_{3}=X_{i} X^{i} \\
\epsilon_{4}=X_{i} Y^{i} \\
\epsilon_{5}=Y_{i} Y^{i}
\end{gathered}
$$

where we have defined the shorthands

$$
\begin{gathered}
X_{i} \equiv f_{i}-\left(b-\frac{\rho}{2}\right) t_{i}+(b-1) d_{i}, \\
Y_{i} \equiv(\log Q)_{, i}+\frac{1}{2}\left(\sigma-\mu_{L}\right) t_{i} .
\end{gathered}
$$

(This energy is found by writing down the most general $\epsilon$ and flux $\phi^{i}$ quadratic in $\gamma_{i j, k}, Q_{, i}, K_{i j}$, and $f_{i}$ constructed using only these and $\gamma^{i j}$, and restricting their $14+10$ free coefficients by 19 conditions arising from energy conservation $\dot{\epsilon}=\phi^{i}{ }_{i}$ in the high-frequency approximation. We do not give the fluxes here. Positive definiteness of $\epsilon$ has not been imposed yet.) Energies $\epsilon_{3}, \epsilon_{4}$, and $\epsilon_{5}$ are auto- matically conserved with zero flux. Conservation of $c_{0} \epsilon_{0}+c_{1} \epsilon_{1}$ requires

$$
\begin{aligned}
c_{0}(1-2 a b+ & \left.\mu_{L}+2 c-2 b d\right) \\
& +2 c_{1}(1-a b+3 c-3 b d)=0,
\end{aligned}
$$

with nonzero flux, and conservation of $\epsilon_{2}$ (with nonzero flux as well) poses no new condition on the parameters. The coefficient $c_{0}$ must be strictly positive and therefore there are two possibilities: either $a b-1 \neq 3(c-b d)$ and then $c_{1}$ is determined by $c_{0}$ and the parameters $(a, b, c, d$, $\left.\mu_{L}\right)$, or we have the special case $a b-1=3(c-b d)=$ $3\left(\mu_{L}-1\right) / 4$ and then $c_{0}$ and $c_{1}$ are independent. Both NOR-A/B belong to that special case and require $\mu_{L}=1$, which is harmonic slicing. Generic NOR however allows for arbitrary values of $\mu_{L}$.

With the evolved lapse we obtain the same slicing as with fixed densitized lapse if we set $\mu_{L}=\sigma$ and $\partial_{t} Q=0$. Then $Y_{i}$ can be dropped from the energy and (125)-(134) reduces to the energy of NOR with densitized lapse and fixed shift (68).

Fixing an overall factor by setting $c_{0}=1$, the energy has either 9 free objects ( 4 coefficients $c_{i}$ plus 5 parameters), or 8 free objects in the special case $(5$ coefficients plus 3 parameters). The ranges for those objects are restricted by the positivity conditions on the energy. The tensor and scalar sectors require

$$
c_{1}>-\frac{1}{3}, \quad-\frac{1}{4}<c_{2}<\frac{1}{2},
$$

and the vector sector requires positivity of a $4 \times 4$ matrix, which is difficult to convert into explicit inequalities for the coefficients and parameters. Some necessary conditions are

$$
c_{3}>0, \quad c_{5}>0, \quad c_{4}^{2}<4 c_{3} c_{5} .
$$

For example for NOR-A with $\mu_{L}=1$ and choosing $c_{4}=0$ we still have 4 free coefficients, and a complete set of symmetric hyperbolicity conditions are

$$
\begin{gathered}
c_{1}>0, \quad 0<c_{2}<\frac{1}{2}, \quad c_{3}>c_{1}+\frac{1}{4 c_{2}}, \\
c_{5}>\frac{\left(4 c_{1} c_{2}+1\right) c_{3}}{4 c_{2}\left(c_{3}-c_{1}\right)-1} .
\end{gathered}
$$

\section{NOR with live lapse and shift}

The most general conserved energy can be written as

$$
\epsilon=\sum_{i=0}^{10} c_{i} \epsilon_{i}
$$

with $\epsilon_{0}, \ldots, \epsilon_{5}$ as given before. Defining 


$$
\begin{aligned}
& W_{i} \equiv \mu_{S}\left[s X_{i}+\left(b s-\frac{\bar{\rho}}{2}\right) t_{i}+(1-b s) \frac{t_{i}}{2}\right] \\
&+\epsilon_{S}\left[Y_{i}+\frac{\mu_{L}}{2} t_{i}\right] \\
& Z_{i} \equiv \mu_{S}\left[s X_{i}+\left(b s-\frac{\bar{\rho}}{2}\right) \frac{t_{i}}{2}\right]+\epsilon_{S}\left[Y_{i}+\frac{\mu_{L}}{4} t_{i}\right],
\end{aligned}
$$

we have 5 new energy terms containing derivatives of the shift vector

$$
\begin{gathered}
\epsilon_{6}=\bar{\beta}_{, j}^{i} \bar{\beta}_{, i}^{j}, \\
\epsilon_{7}=\bar{\beta}_{, i}^{i} \bar{\beta}_{, j}^{j}-\bar{\beta}_{, j}^{i} \bar{\beta}_{, i}^{j}, \\
\epsilon_{8}=\gamma^{i k} \gamma_{j l} \bar{\beta}_{, i}^{j} \bar{\beta}_{, k}^{l}-\bar{\beta}_{, j}^{i} \bar{\beta}_{, i}^{j}, \\
\epsilon_{9}=K_{j}^{i} \bar{\beta}^{j}{ }_{, i}-\frac{\alpha}{2} W^{i} d_{i}, \\
\epsilon_{10}=K \bar{\beta}_{, i}^{i}-\frac{\alpha}{2} Z^{i} t_{i} .
\end{gathered}
$$

Assuming $b \neq 0$ and $\epsilon_{L} \neq \mu_{L}$ (two necessary conditions for strong hyperbolicity in NOR), the coefficients $c_{3}, c_{4}, c_{5}$ are always determined by the rest of the coefficients:

$$
\begin{gathered}
4 b c_{3}=2 a c_{0}+4 s \mu_{S} c_{8}+\left(2 a+s \mu_{S}\right) c_{9}, \\
\left(\epsilon_{L}-\mu_{L}\right) c_{4}=2(a+d) c_{0}+2(a+3 d) c_{1}+2 s \mu_{S} c_{6} \\
+\left(a+d+s \mu_{S}\right) c_{9}+\left(a+3 d+s \mu_{S}\right) c_{10},
\end{gathered}
$$

$$
\left(\epsilon_{L}-\mu_{L}\right) c_{5}=\epsilon_{S} c_{6}-\left(c_{0}+c_{1}\right)+\frac{\epsilon_{S}-1}{2}\left(c_{9}+c_{10}\right) .
$$

Energy conservation imposes 4 more conditions:

$$
\begin{gathered}
\left(a b-\mu_{S}\right) c_{9}+4 \mu_{S}(b s-1) c_{8}=0, \\
2 \epsilon_{L} c_{0}+\left[2 a b-1-2 c+2 b d-\epsilon_{L} \epsilon_{S}+\mu_{S}(\bar{\rho}-2)\right] c_{9} \\
+2(a b-1-3 c+3 b d) c_{10}+4 \mu_{S}(b s-1) c_{6}=0,
\end{gathered}
$$

$$
\begin{aligned}
& {\left[2-2 a b+6 c-6 b d-\epsilon_{L} \epsilon_{S}+\mu_{L}+\mu_{S}(\bar{\rho}-2)\right] c_{10}} \\
& \quad+2\left[\mu_{S}(\bar{\rho}-2 b s)-\epsilon_{S} \mu_{L}\right] c_{6}+2 \epsilon_{L} c_{1} \\
& \quad+\left(1-2 a b+2 c-2 b d+\mu_{L}\right) c_{9}=0, \\
& c_{0}\left(1-2 a b+\mu_{L}+2 c-2 b d\right)+2 c_{1}(1-a b+3 c-3 b d) \\
& \quad+\mu_{S}(1-b s) c_{10}+\frac{1}{2}\left[\mu_{S}(\bar{\rho}-2 b s)-\epsilon_{S} \mu_{L}\right] c_{9}=0 .
\end{aligned}
$$

For generic values of the parameters, these 4 conditions allow us to solve for $c_{6}, c_{8}, c_{9}, c_{10}$, leaving only 4 free coefficients $c_{0}, c_{1}, c_{2}, c_{7}$. It is possible, however, to choose the parameters so that some of those 4 conditions are automatically obeyed, and then the number of free coefficients is larger. For example, for NOR with implicit harmonic gauge and $s=1$ all 4 conditions are automatically obeyed, leaving 8 free coefficients $c_{i}$. Note that the condition (134) has now been transformed into (152). Note also that for $\epsilon_{L}=0$ conditions (149)-(151) only involve the new coefficients $c_{6}, \ldots, c_{10}$.

So far we have only looked at energy conservation. Symmetric hyperbolicity requires positivity of the conserved energy. Ideally, we would like to translate positivity into 2 sets of inequalities: ranges of evolution parameters that lead to a symmetric hyperbolic system, followed by (parameter-dependent) inequalities for the free coefficients of the conserved energy given above. We achieved this for NOR with fixed gauge in [3], but have not managed for the systems studied here.

Sometimes, for example, for the LS system [8], it is possible to choose the coefficients of a generic energy to make it positive, and then solve the energy conservation equations for the evolution parameters of the system. This method proves that the system is symmetric hyperbolic for some values of the parameters, but works only if there are enough parameters in the evolution system. It may also be difficult to find energies relating to specific interesting values of the evolution parameters.

For NOR with LS gauge, we only have 12 parameters, namely, $(a, b, c, d),\left(\mu_{L}, \mu_{S}, \epsilon_{L}, \epsilon_{S}\right)$, and $(\rho, \bar{\rho}, \sigma, s)$, to solve 15 linear equations imposed by energy conservation on 19 coefficients of a general energy. Of the 19 coefficients, 11 multiply squares (diagonal terms) and 8 multiply mixed products (off-diagonal terms). Setting 7 of the offdiagonal terms equal to zero, we have been able to solve the remaining positivity conditions and all energy conservation equations to obtain a 9-parameter (7 evolution parameters and 2 energy coefficients) family of symmetric hyperbolic systems. Three of the 5 relations among the parameters are $\epsilon_{L}=0, b s=1$, and $\mu_{L} \epsilon_{S}=(\bar{\rho}-2) \mu_{S}$; the other 2 are rather longer. However, there is no reason to assume that any of the positivity conditions fail if all offdiagonal coefficients of the energy are given sufficiently small nonzero values. Therefore we believe that our 9parameter family is embedded in a 16-parameter open set of symmetric hyperbolic formulations, which could be expressed in terms of the 12 evolution parameters and 4 energy coefficients.

In particular, we have been able to construct positive and conserved energies for relevant choices of the parameters; for example, for NOR-B with $\mu_{L}=\mu_{S}=s=1, \epsilon_{L}=0$, $\epsilon_{S}=-1$, the 4 conservation equations reduce to $c_{9}(\bar{\rho}-$ $1)=0$ and $\left(2 c_{6}+c_{10}\right)(\bar{\rho}-1)=0$. For $\bar{\rho}=1$ the 8 coefficients are free and a possible positive energy is given by 


$$
\begin{aligned}
& c_{0}=1, \quad c_{1}=112, \quad c_{2}=-\frac{3}{16}, \quad c_{6}=12 \text {, } \\
& c_{7}=-5, \quad c_{8}=16, \quad c_{9}=-8, \quad c_{10}=-8 \text {. }
\end{aligned}
$$

We can also construct a positive energy for BSSN-C ( $\rho=$ $\bar{\rho}=2 / 3, \sigma=0$ ) choosing 6 free coefficients, although we have only been able to solve the inequalities by finding specific sets of energy coefficients, rather than by giving ranges.

\section{HYPERBOLICITY WITH IMPLICIT HYPERBOLIC LAPSE AND EXPLICIT HYPERBOLIC SHIFT DRIVERS}

Recently, Campanelli et al. [19] and Baker et al. [20], followed by Diener et al. [21] and Herrmann et al. [22] have reported significant progress in binary black-hole evolutions with the BSSN-C formulation using an implicit hyperbolic lapse driver combined with an explicit hyperbolic shift driver. The gauge choice differs slightly between these 4 groups. We shall look at a family that includes all 4 cases plus a fifth that we suggest as a simpler alternative. In the following we look at BSSN-C in the form of NOR-B $(a=b=1, c=d=-1 / 3)$ with the further choice $s=$ $1, \rho=\bar{\rho}=2 / 3$, so that $f^{i}=\gamma^{1 / 3} \tilde{\Gamma}^{i}$. We also set $\sigma=0$. The gauge choice we consider is

$$
\begin{gathered}
\left(\partial_{0}+\zeta_{L} \partial_{\bar{\beta}}\right) \ln \alpha \simeq-\mu_{L} K, \\
\left(\partial_{0}+\zeta_{S} \partial_{\bar{\beta}}\right) \beta^{i} \simeq b^{i} \\
\left(\partial_{0}+\zeta_{b} \partial_{\bar{\beta}}\right) b^{i} \simeq \mu_{S} \alpha\left(\partial_{0}+\zeta_{f} \partial_{\bar{\beta}}\right) \bar{f}^{i} .
\end{gathered}
$$

(In each case, the factor of proportionality between our variable $b^{i}$ and the variable $B^{i}$ in which these gauges are originally formulated is different. We have introduced $b^{i}$ to simplify the comparison of the principal terms.) In the principal part, this corresponds to the gauge choices of the 4 groups with the values of the 6 parameters given in Table I. All 4 groups set $\zeta_{S}=\zeta_{b}=1$. By eliminating the variable $b^{i}$ in favor of a second time derivative of $\beta^{i}$, and noting that $b^{i}$ does not appear anywhere else in the evolu- tion equations, we see that $\left(\beta_{S}, \beta_{b}\right)=(0,1)$ and $(1,0)$ would be equivalent in the principal part.

With the shorthands

$$
\begin{gathered}
\lambda_{1 \pm} \equiv-\frac{\bar{\beta}_{n}}{2} \pm \sqrt{\left(\frac{\bar{\beta}_{n}}{2}\right)^{2}+\mu_{L}} \\
\lambda_{2 \pm} \equiv-\frac{\bar{\beta}_{n}}{2} \pm \sqrt{\left(\frac{\bar{\beta}_{n}}{2}\right)^{2}+\frac{4}{3} \mu_{S}} \\
\lambda_{3 \pm} \equiv-\bar{\beta}_{n} \pm \sqrt{\frac{4}{3} \mu_{S}} \\
\lambda_{4 \pm} \equiv-\frac{\bar{\beta}_{n}}{2} \pm \sqrt{\left(\frac{\bar{\beta}_{n}}{2}\right)^{2}+\mu_{S}}
\end{gathered}
$$

the second column of Table I then gives all characteristic speeds of these 4 systems. In all 4 systems strong hyperbolicity breaks down when some of the speeds coincide. The conditions for avoiding this are listed in the third column of the table. It is possible that these inequalities hold for the specific binary black-hole evolutions because $\bar{\beta}^{i}$ was sufficiently small in them, but one would expect numerical difficulties to appear for $\bar{\beta}^{i}$ sufficiently large to violate one of these conditions, for example, in corotating coordinates. It would be simple and interesting to verify this.

If we set $\zeta_{L}=\zeta_{S}=\zeta_{b}=\zeta_{f}=0$, all speeds become independent of $\bar{\beta}^{i}$. They are

$$
\left(0, \pm 1, \pm \sqrt{\mu_{L}}, \pm \sqrt{\mu_{S}}, \pm \sqrt{4 \mu_{S} / 3}\right)
$$

and the conditions for strong hyperbolicity are $\mu_{S} \neq \mu_{L}$ and $\mu_{S} \neq 3 \mu_{L} / 4$, which can now easily be arranged through the choice of $\mu_{L}$ and $\mu_{S}$ as functions of $\alpha$, for example $\mu_{S}=3 / 4$ and $\mu_{L}=2 / \alpha$, which would not coincide as long as $\alpha<2$, independently of the shift. Strong hyperbolicity of BSSN with this gauge choice was shown by Beyer and Sarbach [23]. Hyperbolicity of the Z4 formulation with a family of gauges with the same principal part and, in our notation, $\zeta_{f}=\zeta_{S}=0$ and $\zeta_{L}$ and $\zeta_{b}$ free, was examined in [24].

TABLE I. Parameter values, characteristic speeds, and conditions for strong hyperbolicity for the 4 "puncture evolution" codes and the variant of Beyer and Sarbach. For comparison with these authors, we have expressed our parameters $\mu_{L}$ and $\mu_{S}$ in their notation, but for comparison between the gauges we give the characteristic speeds in our notation.

\begin{tabular}{lcccccccc}
\hline \hline & $\zeta_{L}$ & $\zeta_{S}$ & $\zeta_{b}$ & $\zeta_{f}$ & $\mu_{L}$ & $\mu_{S}$ & Speeds & Strongly hyperbolic for \\
\hline Campanelli et al. & 0 & 1 & 1 & 1 & $\frac{2}{\alpha}$ & $\frac{3}{4} \alpha^{-2} \gamma^{1 / 3}$ & $\left(0, \pm 1, \pm \sqrt{\mu_{L}},-\bar{\beta}_{n}, \lambda_{2 \pm}, \pm \sqrt{\mu_{S}}\right)$ & $|\bar{\beta}|<\left|\left(3 \mu_{L}-4 \mu_{S}\right) /\left(3 \sqrt{\mu_{L}}\right)\right|,|\bar{\beta}|<\sqrt{\mu_{S}}$ \\
Baker et al. & 0 & 1 & 1 & 0 & $\frac{2}{\alpha}$ & $\frac{3}{4} \alpha^{-1} \gamma^{1 / 3}$ & $\left(0, \pm 1, \pm \sqrt{\mu_{L}}, \lambda_{3 \pm}, \lambda_{4 \pm}\right)$ & $|\bar{\beta}|<\sqrt{4 \mu_{S} / 3},|\bar{\beta}|<\left|\sqrt{\mu_{L}}-\sqrt{4 \mu_{S} / 3}\right|$ \\
Diener et al. & 1 & 1 & 1 & 1 & $\frac{2 \psi_{\mathrm{BL}}^{m}}{\alpha}$ & $\frac{3}{4} \frac{\alpha^{p}-2}{\psi_{\mathrm{BL}}^{n}} \gamma^{1 / 3}$ & $\left(0, \pm 1,-\bar{\beta}_{n}, \lambda_{1 \pm}, \lambda_{2 \pm}, \pm \sqrt{\mu_{S}}\right)$ & $\mu_{L} \neq 4 \mu_{S} / 3,|\bar{\beta}|<\sqrt{\mu_{S}}$ \\
Herrmann et al. & 1 & 1 & 1 & 0 & $\frac{2}{\alpha}$ & $\frac{3}{4} \alpha^{-2} \gamma^{1 / 3}$ & $\left(0, \pm 1,-\bar{\beta}_{n}, \lambda_{1 \pm}, \lambda_{3 \pm}, \lambda_{4 \pm}\right)$ & $|\bar{\beta}|<\left|\left(3 \mu_{L}-4 \mu_{S}\right) /\left(2 \sqrt{3 \mu_{S}}\right)\right|$ \\
Beyer-Sarbach & 0 & 0 & 0 & 0 & $f$ & $G H$ & $\left(0, \pm 1, \pm \sqrt{\mu_{L}}, \pm \sqrt{\mu_{S}}, \pm \sqrt{4 \mu_{S} / 3}\right)$ & $\mu_{S} \neq \mu_{L}, \mu_{S} \neq 3 \mu_{L} / 4$ \\
\hline \hline
\end{tabular}




\section{MODE ANALYSIS RESULTS}

\section{A. Formalism}

If the complete evolution system is not hyperbolic, we can use mode analysis to obtain at least necessary conditions for well-posedness. We make two approximations: we linearize around a background, and we approximate all background-dependent coefficients of the linearized equations as constant ("frozen"). Physically this corresponds to investigating small amplitude, high-frequency perturbations. (The results obtained will in general depend both on the background solution, and the choice of gauge on it.)

In the resulting linear problem with constant coefficients we take a Fourier transform

$$
u\left(x^{i}, t\right) \equiv \int e^{i \omega_{i} x^{i}} \hat{u}\left(\omega_{i}, t\right) d^{3} \omega
$$

and consider the evolution of one Fourier mode at a time. Well-posedness of the Cauchy problem means that $\|u(\cdot, t)\| \leq f(t)\|u(\cdot, 0)\|$ with $f(t)$ independent of the initial data $u\left(x^{i}, 0\right)$. This is the case if and only if a similar bound can be obtained for $\hat{u}\left(\omega_{i}, t\right)$ with $f(t)$ independent of $\omega_{i}$. To establish well-posedness it is sufficient to consider only certain leading order terms. This is well-known for strongly hyperbolic first-order systems [25], but we need a generalization which is given in the following:

Theorem 1: Consider a linear problem with constant coefficients which in pseudodifferential form is

$$
\begin{gathered}
\partial_{t} \hat{u}\left(\omega_{i}, t\right)=\left[M\left(\omega_{i}\right)+M^{\prime}\left(\omega_{i}\right)\right] \hat{u}\left(\omega_{i}, t\right), \\
\hat{u}\left(\omega_{i}, 0\right)=\hat{u}_{0}\left(\omega_{i}\right) .
\end{gathered}
$$

The initial-value problem is well-posed if $M\left(\omega_{i}\right)$ is diagonalizable,

$$
M\left(\omega_{i}\right)=T^{-1}\left(\omega_{i}\right) \Lambda\left(\omega_{i}\right) T\left(\omega_{i}\right)
$$

with $\Lambda\left(\omega_{i}\right)$ diagonal, and if $M\left(\omega_{i}\right)$ and $M^{\prime}\left(\omega_{i}\right)$ obey the bounds

$$
\begin{gathered}
\left|T\left(\omega_{i}\right)\right|\left|T^{-1}\left(\omega_{i}\right)\right| \leq K_{1}, \\
\left|\operatorname{Re} \Lambda\left(\omega_{i}\right)\right| \leq K_{2}, \\
\left|M^{\prime}\left(\omega_{i}\right)\right| \leq K_{3},
\end{gathered}
$$

with $K_{1}, K_{2}$, and $K_{3}$ independent of $\omega_{i}$.

For a more general statement in the context of semigroup theory, see [26]. An elementary proof of our version of the theorem is given in Appendix A, and a technical complication in applying the theorem to second order in space systems is resolved in Appendix B.

Clearly the well-posedness of the linearized, frozencoefficients problem is necessary for the well-posedness of the full problem. For strongly hyperbolic problems wellposedness of the linearized frozen-coefficient problem around any background, together with smoothness of
$T\left(\omega_{i}\right)$, is also sufficient [25], but we do not know if that is true for the wider class of problems considered here.

\section{B. Implicit hyperbolic drivers}

To establish notation we recast the problem of strong hyperbolicity of the NOR formulation with implicit hyperbolic lapse and shift drivers, which was already discussed in Section IV, into the language of pseudodifferential operators. We define

$$
\begin{gathered}
\hat{u} \equiv(i \omega \hat{v}, \hat{w}), \\
\hat{Z} \equiv i \omega \hat{z},
\end{gathered}
$$

where $\omega \equiv \sqrt{\gamma^{i j} \omega_{i} \omega_{j}}$ and $z=\left(\ln \alpha, \beta^{i}\right)$. The factors $i \omega$ appear because this is a pseudodifferential reduction to first order of a second-order in space system [6]. We define the pseudodifferential equivalent of the derivative operator $\partial_{0}$,

$$
\hat{\partial}_{0} \equiv \alpha^{-1}\left(\partial_{t}-i \omega \beta_{n} I\right) \text {. }
$$

The evolution of the gauge variables has the principal part $\dot{z} \simeq w+\partial v+\partial z$, and combining this with (2) and (3) the coupled pseudodifferential equations are

$$
\hat{\partial}_{0}\left(\begin{array}{c}
\hat{u} \\
\hat{z}
\end{array}\right) \simeq i \omega\left(\begin{array}{cc}
P & Q \\
V & W
\end{array}\right)\left(\begin{array}{c}
\hat{u} \\
\hat{Z}
\end{array}\right)
$$

where $P, Q, V$, and $W$ are independent of $\omega$ but depend on $n_{i} \equiv \omega_{i} / \omega$. Here $\simeq$ means that terms corresponding to the lower-order part $M^{\prime}\left(\omega_{i}\right)$ have been neglected. (In the context of a pseudodifferential reduction to first order there is a slight complication in the definition of $M^{\prime}\left(\omega_{i}\right)$, which is discussed in Appendix B.) $P$ and $Q$ can be read off from (20)-(22). The nontrivial part of the matrix $V$ is given by (89). $W$ contains the parameters $\zeta_{L}, \zeta_{S}, \epsilon_{L}$, and $\epsilon_{S}$ defined in (111) and (112).

We have analyzed this system above in Sec. IV, and do not discuss it further here.

\section{Explicit parabolic drivers}

These have the general form $\dot{z} \simeq \dot{w}+\partial \dot{v}+\partial \partial z$. The pseudodifferential form of the coupled equations is

$$
\hat{\partial}_{0}\left(\begin{array}{c}
\hat{u} \\
\hat{Z}
\end{array}\right) \simeq\left(\begin{array}{cc}
i \omega P & i \omega Q \\
-\omega^{2} R+i \omega R^{\prime} & -\omega^{2} S+i \omega S^{\prime}
\end{array}\right)\left(\begin{array}{l}
\hat{u} \\
\hat{Z}
\end{array}\right),
$$

where the matrices $R, S, R^{\prime}$, and $S^{\prime}$ are independent of $\omega$. Note that in order to apply Thm. 1 we need to work with the lower-order terms $R^{\prime}$ and $S^{\prime}$. To calculate them we need to explicitly linearize the gauge conditions. If the principal part matrix defined in (174) obeys the conditions of Thm. 1, we obtain a necessary condition for wellposedness in $L^{2}(w, \partial v, \partial z)$. Alternatively, we can use a different pseudodifferential reduction of the form 


$$
\hat{\partial}_{0}\left(\begin{array}{c}
\hat{u} \\
\hat{z}
\end{array}\right) \simeq\left(\begin{array}{cc}
i \omega P & -\omega^{2} Q+i \omega Q^{\prime} \\
i \omega R & -\omega^{2} S+i \omega S^{\prime}
\end{array}\right)\left(\begin{array}{c}
\hat{u} \\
\hat{z}
\end{array}\right) .
$$

The conditions for Thm. 1 are then a necessary condition for well-posedness in $L^{2}(w, \partial v, z)$. The lower-order terms that must be kept are now $S^{\prime}$ and $Q^{\prime}$.

When either set of lower-order terms is kept, the resulting principal part matrix $M\left(\omega_{i}\right)$ becomes very complicated. In particular, the eigenvalues depend on the background spacetime, and the matrix no longer splits into block-diagonal form with scalar, vector, and tensor blocks. In order to make progress, we linearize around Minkowski spacetime using Cartesian coordinates.

In the Cartesian Minkowski case the lower-order terms $R^{\prime}, S^{\prime}$, and $Q^{\prime}$ all vanish, and we are left with the simpler matrices $M\left(\omega_{i}\right)$ of the form

$$
\left(\begin{array}{cc}
i \omega P & i \omega Q \\
-\omega^{2} R & -\omega^{2} S
\end{array}\right) \text { or }\left(\begin{array}{cc}
i \omega P & -\omega^{2} Q \\
i \omega R & -\omega^{2} S
\end{array}\right)
$$

which are similar, and therefore equivalent for the purposes of Thm. 1. The diagonalizability and eigenvalues of these matrices already give interesting necessary conditions for well-posedness.

We consider explicit parabolic drivers in the form

$$
\begin{aligned}
& \partial_{0} \ln \alpha \simeq-\nu_{L} \partial_{0} K, \\
& \partial_{0} \beta^{i} \simeq-\nu_{S} \alpha \partial_{0} \bar{f}^{i} .
\end{aligned}
$$

Note that when only principal terms are considered we can pull the factor of $\alpha$ out of the time derivative in (178). $R$ and $S$ can then be read off from (84) and (91). We find that the simplified necessary conditions based on linearization around Minkowski are obeyed for this gauge with ADM and NOR-B, but not NOR-A (the scalar sector fails to be diagonalizable), for $\bar{\rho}<2, \nu_{L}>0, \nu_{S}>0$, and $\nu_{L} \neq(2-$ $\bar{\rho}) \nu_{S}$. The eigenvalues are $0, \pm i \omega,-\nu_{L} \omega^{2}$, and $-(2-$ $\bar{\rho}) \nu_{S} \omega^{2}$.

The lapse condition (177) with fixed shift together with ADM or NOR-A/B is ill-posed according to Thm. 1. This can be understood roughly as follows: The principal part of the lapse evolution equation is $\dot{\alpha} \sim \Delta \alpha$, which is autonomous. $\alpha$ can therefore be considered as a given function in the principal part of the remaining evolution equations, and so their principal part is the same as for fixed (undensitized) lapse (and fixed shift).

To investigate the explicit parabolic drivers using $\partial_{t}$ on both the left and right-hand sides, that is, (92) and (96), we consider the matrix

$$
\left(\begin{array}{cc}
i \omega P & i \omega Q \\
-\omega^{2} R+i \omega \bar{\beta}_{n} T & -\omega^{2} S-i \omega \bar{\beta}_{n} I
\end{array}\right) .
$$

(Again we drop the lower-order terms by linearizing around Minkowski.) Neither ADM or NOR-A/B are diagonalizable with this gauge, except of course for $\bar{\beta}_{n}=0$.
Because the explicit parabolic lapse driver with fixed shift using $\partial_{0}$ alone is not well-posed (as we saw above), neither is the version using $\partial_{t}$ as the two coincide for $\bar{\beta}_{n}=$ 0 .

\section{Implicit parabolic drivers}

These have the general form $z \simeq w+\partial v$. After linearization, in pseudodifferential form

$$
\hat{Z} \simeq\left(i \omega T+T^{\prime}\right) \hat{u},
$$

where $T$ and $T^{\prime}$ are independent of $\omega$. In order to calculate the lower-order term $T^{\prime}$, we again need to explicitly linearize the gauge conditions. Eliminating $\hat{Z}$, we find

$$
\hat{\partial}_{0} \hat{u} \simeq\left[-\omega^{2} Q T+i \omega\left(P+Q T^{\prime}\right)\right] \hat{u} .
$$

We can again make progress by linearizing around Minkowski spacetime in standard coordinates, so that $T^{\prime}$ vanishes. $T$ is equal to $V$ with $\mu_{L}$ and $\mu_{S}$ replaced by $\nu_{L}$ and $\nu_{S}$.

The conditions of Thm. 1 are obeyed for the implicit parabolic drivers (93) and (97) with ADM and NOR, with the same inequality conditions as for the explicit parabolic drivers. The eigenvalues are also the same as for the explicit parabolic drivers.

The implicit parabolic lapse driver (93) with fixed shift obeys the conditions of Thm. 1 for NOR-B for $\nu_{L}>0$. The eigenvalues are $0, \pm i \omega$, and $-\nu_{L} \omega^{2}$. This is not true for NOR-A (because the scalar sector is not diagonalizable) or ADM (because the vector sector is not diagonalizable).

\section{E. Elliptic freezing conditions}

These have the general form $\dot{w}+\partial \dot{v} \simeq 0$. After linearization, in pseudodifferential form,

$$
0 \simeq\left(-\omega^{2} R+i \omega R^{\prime}\right) \hat{u}+\left(-\omega^{2} S+i \omega S^{\prime}\right) \hat{Z},
$$

where $R^{\prime}, R, S^{\prime}$, and $S$ can be chosen to be the same matrices as above. Eliminating $\hat{Z}$, we obtain the reduced system

$$
\hat{\partial}_{0} \hat{u} \simeq i \omega\left(P-Q S^{-1} R\right) \hat{u} .
$$

Note that this algebraic reduction can only be carried out in pseudodifferential form. Intuitively speaking, $R^{\prime}$ and $S^{\prime}$ do not appear in the principal part because of the smoothing effect of solving an elliptic equation.

The elliptic freezing conditions $\partial_{0} K=0, \partial_{0} \bar{f}^{i}=0$ obey the conditions of Thm. 1 together with ADM and NOR-B but not NOR-A (the scalar sector is not diagonalizable) for $\bar{\rho} \neq 2$. The speeds are 0 and \pm 1 .

The elliptic lapse condition alone, with fixed shift, is not diagonalizable for either of the three systems. This slightly surprising result can be explained as follows: The only component of $\partial_{0} K_{i j} \simeq-(\ln \alpha)_{, i j}+\ldots$ in the pseudodifferential approach is $\hat{\partial}_{0} \hat{K}_{n n} \simeq \omega^{2}(\hat{\ln \alpha})+\ldots$. But the elliptic equation for $\alpha$ is $-\omega^{2}(\hat{\ln \alpha}) \simeq 0$. The scalar sector of the 
principal part is then the same as for fixed (undensitized) lapse, and so is not diagonalizable.

However, if one formally enforces the constraint $K=$ $\phi(x)$ by setting $\hat{K}_{q q} \simeq-\hat{K}_{n n}$ the scalar sector of NOR-A/B (but not ADM) becomes diagonalizable, and the resulting system obeys the conditions of Thm. 1. In the BSSN formulation $K$ is an explicit variable, and one can enforce $K=\phi(x)$ by not evolving this variable when BSSN is used with maximal slicing and fixed shift [27]. With this modification the system is well-posed, but if $K$ was evolved it would be ill-posed.

\section{F. Implicit hyperbolic drivers with parabolic damping terms}

The gauge conditions (105) and (108), linearized around Minkowski in Cartesian coordinates, give

$$
\hat{\partial}_{0}\left(\begin{array}{c}
\hat{u} \\
\hat{Z}
\end{array}\right) \simeq\left(\begin{array}{cc}
i \omega P & i \omega Q \\
i \omega V-2 \omega^{2} R & i \omega W-2 \omega^{2} S
\end{array}\right)\left(\begin{array}{l}
\hat{u} \\
\hat{Z}
\end{array}\right) .
$$

For simplicity we restrict the hyperbolic gauge conditions to $\zeta=\epsilon_{L}=\epsilon_{S}=0$, and we consider ADM. Without the damping terms, that is with $\nu_{L}=\nu_{S}=0$, the system is strongly hyperbolic for $\mu_{L}>0, \mu_{S}>0$, and $\bar{\rho}<2$, with eigenvalues $0, \pm i \omega, \pm i \omega \sqrt{\mu_{L}}, \pm i \omega \sqrt{(2-\bar{\rho}) \mu_{S}}$, and $\pm i \omega \sqrt{\mu_{S}}$, provided that $\mu_{L} \neq 1, \mu_{S} \neq 1 /(2-\bar{\rho})$, and $\mu_{S} \neq \mu_{L} /(2-\bar{\rho})$, corresponding to real speeds. With the damping turned on, the pair of eigenvalues $\pm i \omega \sqrt{\mu_{L}}$ becomes

$$
-\omega^{2} \sqrt{\nu_{L}} \pm \sqrt{\omega^{4} \nu_{L}^{2}-\omega^{2} \mu_{L}},
$$

and similarly for the other eigenvalues. This has negative real part for all $\omega>0$. However, the scalar sector fails to be diagonalizable if these two eigenvalues coincide, that is for $\omega=\sqrt{\mu_{L}} / \nu_{L}$. Similar problems arise for $\omega=$ $\sqrt{\mu_{L}} /\left(\sqrt{2-\bar{\rho}} \nu_{L}\right)$ in the scalar sector and $\omega=\sqrt{\mu_{L}} / \nu_{L}$ in the vector sector. In numerical applications, choosing $\nu_{S}, \nu_{L} \propto \Delta x$ with a sufficiently small constant of proportionality makes the problematic wave numbers larger than the grid frequency, and this ill-posedness should then not lead to numerical problems [17]. Similar results hold for NOR-A/B at least in the special cases $s=0$ or $\rho=2$.

\section{CONCLUSIONS}

Continuing our research programme on second order in space, first order in time formulations of the Einstein equations $[3,6,28]$, we have reviewed the relationship between the BSSN formulation of the Einstein equations that is widely used in numerical relativity, and the NOR formulation (NOR-A) that was suggested as a simpler alternative to BSSN. We have showed that a variant of NOR, (NOR-B) has the same principal part as BSSN when the latter is formally restricted to solutions of the algebraic constraints (BSSN-C).
We have shown that the principal parts of the KST formulation and the NOR formulation are equivalent when the gauge is fixed. Each formulation has five parameters, and we have given four relations between the two sets of parameters. One parameter in each formulation does not have a counterpart in the other formulation and does not influence the level of hyperbolicity. The analogy also holds with an evolved lapse, and symmetric hyperbolic cases exist for both fixed gauge and live lapse. With an evolved lapse and shift, KST has 4 parameters more than NOR with the same gauge.

We have reviewed various differential equations for the lapse and shift currently in use as coordinate conditions in numerical relativity, with an emphasis of their origin as symmetry-seeking coordinates, and their classification as elliptic, parabolic, hyperbolic, or neither. These are all based on $K$-freezing shift and $\Gamma$-freezing lapse, which in turn are related to the well-known maximal slicing, minimal distortion gauge.

From the point of view of "symmetry-seeking" gauge conditions it is more natural to have evolution equations for the gauge of the form $\partial_{t} \alpha, \partial_{t} \beta=\ldots$, while the ADM evolution equations are naturally of the form $\partial_{0} \gamma_{i j}, \partial_{0} K_{i j}=\ldots$ With this mixture, there are two sets of characteristic cones in the system, one centered around $n^{a}$ and one around $(\partial / \partial t)^{a}$. Where these overlap, there is a danger of strong hyperbolicity breaking down, but we have shown that some $\partial_{t}$ gauges can be implemented in $\partial_{0}$ form, which would avoid this problem.

We have analyzed the hyperbolicity of the ADM, NORA, and NOR-B formulations with the most general implicit hyperbolic lapse and shift drivers. Interestingly, NOR-A and NOR-B can be made strongly hyperbolic even when using some live gauges with $\partial_{t}$.

We have also investigated the hyperbolicity of NOR-B (equivalent to BSSN-C) with a family of implicit hyperbolic lapse and explicit hyperbolic shift drivers that includes the gauges used by 4 different research groups in "moving puncture" evolutions. We find that all these gauges become ill-posed for large enough values of the shift, and propose a simple modification (replacing $\partial_{t}$ by $\partial_{0}$ ) that is strongly hyperbolic for arbitrary shift.

For certain families of elliptic gauge conditions, parabolic drivers, and hyperbolic drivers with a heat equationtype damping, where the notion of hyperbolicity does not apply, we have carried out the mode analysis and have checked a necessary condition for well-posedness of the Cauchy problem.

Khoklov and Novikov [29] have investigated the wellposedness of a number of gauges independently of any particular formulation in the frozen-coefficient approximation. Their well-posedness is therefore a necessary condition for ours. We review their method and compare results in Appendix C.

Our results for puncture gauges are summarized in Table I and our results for all other gauges in Table II. 
TABLE II. Overview of well-posedness results for combinations of gauge conditions and formulations of the Einstein equations. In the first two rows, strong hyperbolicity has been checked. In the other rows, only a necessary condition (Thm. 1) has been checked. A question mark indicates that we have not done the calculation.

\begin{tabular}{lcc}
\hline \hline Type & Lapse + shift & Lapse only \\
\hline impl. hyp. using $\partial_{0}$ & ADM, NOR-A/B & NOR-A/B \\
impl. hyp. using $\partial_{t}$ & NOR-A/B & NOR-B \\
expl. par. using $\partial_{0}$ & ADM, NOR-B & no \\
expl. par. using $\partial_{t}$ & no & no \\
implicit parabolic & ADM, NOR-A/B & NOR-B \\
elliptic & ADM, NOR-B & no \\
impl. hyp. using $\partial_{0}$ with par. damping & ADM, NOR-A/B & $?$ \\
\hline \hline
\end{tabular}

They are positive for most combinations of gauge and formulation, with a few interesting exceptions. They provide a theoretical underpinning to some conditions already in use in numerical relativity, and suggest certain improvements.

All equations in Sec. IV were derived using $x$ Tensor, an open-source Mathematica package for abstract tensor calculations, developed by J.M. M. It is available under the GNU Public License from http://metric.iem.csic.es/ Martin-Garcia/xAct.

\section{ACKNOWLEDGMENTS}

We would like to thank G. Calabrese, L. Lehner, L. Lindblom, and O. Sarbach for helpful discussions. J.M.M. was supported by the Spanish MEC under the research project No. FIS2005-05736-C03-02.

\section{APPENDIX A: PROOF OF THEOREM 1}

We go to the diagonal basis $\hat{U}=T \hat{u}$. It obeys

$$
\partial_{t} \hat{U}=\left(\Lambda+T M^{\prime} T^{-1}\right) \hat{U},
$$

and so its norm squared obeys

$$
\partial_{t}\left(\hat{U}^{\dagger} \hat{U}\right) \leq 2\left(K_{2}+K_{1} K_{3}\right) \hat{U}^{\dagger} \hat{U} .
$$

This can be integrated to give

$$
\left|\hat{U}\left(\omega_{i}, t\right)\right| \leq e^{\left(K_{2}+K_{1} K_{3}\right) t}\left|\hat{U}\left(\omega_{i}, 0\right)\right|,
$$

and going back to the original basis we have

$$
\left|\hat{u}\left(\omega_{i}, t\right)\right| \leq K_{1} e^{\left(K_{2}+K_{1} K_{3}\right) t}\left|\hat{u}\left(\omega_{i}, 0\right)\right|,
$$

that is, the growth is bounded independently of the wave number $\omega$. Taking the inverse Fourier transform and using Parseval's Theorem, we obtain the $L^{2}$ estimate

$$
\|u(\cdot, t)\| \leq K_{1} e^{\left(K_{2}+K_{1} K_{3}\right) t}\|u(\cdot, 0)\|
$$

in real space.

The well-known result that lower-order terms do not affect the well-posedness of strongly hyperbolic systems (see, for example, Theorem 4.3.2 of [25]) is a special case of Thm. 1. A system of linear first-order evolution equations is strongly hyperbolic if $M\left(\omega_{i}\right)=i \omega_{i} P^{i} \equiv i \omega P_{n}$, with $\omega_{i} \equiv \omega n_{i}$ and $P_{n} \equiv n_{i} P^{i}$, and where $P_{n}$ is diagonalizable with real eigenvalues for every $n_{i}$ (so that $\operatorname{Re} \Lambda=0$ ), $T$ depends smoothly on $n_{i}$ but not on $\omega$ (and so is bounded), and $M^{\prime}$ is independent of $\omega_{i}$ (and so is bounded).

\section{APPENDIX B: PSEUDODIFFERENTIAL REDUCTION TO FIRST ORDER}

The pseudodifferential form of the linearization of (2) and (3) on a constant background, neglecting the terms in $z$ and writing all (linearized) lower-order terms, is

$$
\hat{\partial}_{0}\left(\begin{array}{c}
\hat{v} \\
\hat{w}
\end{array}\right) \simeq\left(\begin{array}{cc}
i \omega A+A^{\prime} & B \\
-\omega^{2} C+i \omega C^{\prime}+C^{\prime \prime} & i \omega D+D^{\prime}
\end{array}\right)\left(\begin{array}{c}
\hat{v} \\
\hat{w}
\end{array}\right),
$$

where the matrices $A, A^{\prime}$, etc. are independent of $\omega$ but depend on $n_{i}$. Replacing $\hat{v}$ by $\hat{V} \equiv i \omega \hat{v}$ multiplies the first row by $i \omega$ and divides the first column by $i \omega$, so that the highest power of $\omega$ is the first power. This constitutes a pseudodifferential reduction to first order.

The terms proportional to $i \omega$ form $M\left(\omega_{i}\right)$ and the terms of $O(1)$ go into $M^{\prime}\left(\omega_{i}\right)$. The term $(i \omega)^{-1} C^{\prime \prime}$ poses a problem, as it is not bounded independently of $\omega_{i}$. Intuitively, it should be part of $M^{\prime}\left(\omega_{i}\right)$ because we are interested mainly in the limit $\omega \rightarrow \infty$. We can obtain a rigorous result to the same effect if we retain $\hat{v}$ in the system. We then have

$$
\hat{\partial}_{0}\left(\begin{array}{c}
\hat{v} \\
\hat{V} \\
\hat{w}
\end{array}\right) \simeq i \omega\left(\begin{array}{ccc}
0 & 0 & 0 \\
0 & A & B \\
0 & C & D
\end{array}\right)\left(\begin{array}{c}
\hat{v} \\
\hat{V} \\
\hat{w}
\end{array}\right)+\left(\begin{array}{ccc}
A^{\prime} & A & B \\
0 & A^{\prime} & 0 \\
C^{\prime \prime} & C^{\prime} & D^{\prime}
\end{array}\right)\left(\begin{array}{c}
\hat{v} \\
\hat{V} \\
\hat{w}
\end{array}\right) .
$$

For the purposes of Thm. 1 we only need to analyze $M\left(\omega_{i}\right)$, which here only means the block $((A, B),(C, D))$, which is called $P$ in the main text.

\section{APPENDIX C: ANALYSIS OF PURE GAUGE PERTURBATIONS}

The effect of a set of gauge conditions can be evaluated in purely geometric terms, independently of any formula- 
tion of the Einstein equations. In this approach, one considers a fixed spacetime obeying all 10 Einstein equations. One evolves only the 4 coordinates $x^{\mu}$ and the vector $(\partial / \partial t)^{a}$ on this spacetime background, treating $x^{\mu}$ as 4 scalar fields and $(\partial / \partial t)^{a}$ as a vector field on the background spacetime. To make connection with the $3+1$ split, let $n^{a}$ be the unit normal on the surfaces of constant $x^{0}$, and let $(\partial / \partial t)^{a}=\alpha n^{a}+\beta^{a}$, where $\beta^{a} n_{a} \equiv 0$. The resulting nonlinear evolution system for $\left(x^{\mu}, \alpha, \beta^{i}\right)$ and a numerical implementation are described in [30].

A linearization of this scheme is used in [29] to investigate the well-posedness of gauges. The gauge perturbation is parametrized by a vector field $\psi_{a}$, which gives rise to a perturbation of the 4-metric $\delta g_{a b}=-2 \nabla_{(a} \psi_{b)}$. The 10 components of this equation can be written in a $3+1$ split as

$$
\begin{gathered}
\dot{\psi}_{0}=\alpha \delta \alpha-\beta^{i} \delta \beta_{i}-\beta^{i} \beta^{j} \psi_{, i j}+\left(\Gamma^{\mu}{ }_{00}+\beta^{i} \beta^{j} \Gamma^{\mu}{ }_{i j}\right) \psi_{\mu}, \\
\dot{\psi}_{i}=-\delta \beta_{i}-\psi_{0, i}+2 \Gamma^{\mu}{ }_{i 0} \psi_{\mu}, \\
\delta \gamma_{i j}=-2 \psi_{(i, j)}+2 \Gamma^{\mu}{ }_{i j} \psi_{\mu} .
\end{gathered}
$$

$\delta K_{i j}$ can be obtained by taking a time derivative of (C3) and using the definition of the extrinsic curvature tensor (5). These equations are coupled with algebraic, elliptic, or evolution equations for $\delta \alpha$ and $\delta \beta_{i}$ in terms of $\delta \gamma_{i j}$ and $\delta K_{i j}$. Clearly the well-posedness of any gauge defined in this way is a necessary condition for that gauge to be wellposed together with any specific formulation of the Einstein equations.

[29] shows that the combination $\dot{\alpha}=\epsilon \dot{K}, \beta^{i}=0$ (explicit parabolic $K$-driver with zero shift) is ill-posed. By taking the limit $\epsilon=0$, it is also claimed $K$-freezing lapse with zero shift is ill-posed, although this limit is singular. Our results agree with these claims. However, we only consider the linearization around Minkowski spacetime in Cartesian coordinates, which simplifies the calculation, while in [29] all lower-order terms in an explicit linearization are kept, and are found to give an unbounded $\operatorname{Re} \Lambda$ for the parabolic lapse driver with fixed shift. On the other hand we find that $M\left(\omega_{i}\right)$ is not diagonalizable when perturbing around the Minkowski background, while diagonalizability is not checked in [29].
[1] J.W. York, Jr., in Sources of Gravitational Radiation, edited by L. Smarr (Cambridge University Press, Cambridge, England, 1979).

[2] G. Nagy, O.E. Ortiz, and O. A. Reula, Phys. Rev. D 70, 044012 (2004).

[3] C. Gundlach and J. M. Martín-García, Phys. Rev. D 70, 044032 (2004).

[4] H.-J. Yo, T. W. Baumgarte, and S. L. Shapiro, Phys. Rev. D 66, 084026 (2002).

[5] I. Hinder, Ph.D. thesis, University of Southampton, 2005.

[6] C. Gundlach and J.M. Martín-García, gr-qc/0506037 [Class. Quant. Grav. (to be published)].

[7] L. E. Kidder, M. A. Scheel, and S. A. Teukolsky, Phys. Rev. D 64, 064017 (2001).

[8] L. Lindblom and M. A. Scheel, Phys. Rev. D 67, 124005 (2003).

[9] O. Sarbach et al., Phys. Rev. D 66, 064002 (2002).

[10] O. Sarbach and M. Tiglio, Phys. Rev. D 66, 064023 (2002).

[11] D. Garfinkle and C. Gundlach, Classical Quantum Gravity 16, 4111 (1999).

[12] L. Smarr and J. W. York, Jr., Phys. Rev. D 17, 2529 (1978).

[13] S. Bonazzola et al., Phys. Rev. D 70, 104007 (2004).

[14] J. Balakrishna et al., Classical Quantum Gravity 13, L135
(1996).

[15] B. Reimann et al., Phys. Rev. D 71, 064021 (2005).

[16] M. Alcubierre et al., Phys. Rev. D 67, 084023 (2003).

[17] M. Shibata, K. Taniguchi, and K. Uryu, Phys. Rev. D 68, 084020 (2003).

[18] L. Lindblom (personal communication).

[19] M. Campanelli et al., Phys. Rev. Lett. 96, 111101 (2006).

[20] J. G. Baker et al., Phys. Rev. Lett. 96, 111102 (2006).

[21] P. Diener et al., Phys. Rev. Lett. 96, 121101 (2006).

[22] F. Herrmann et al., gr-qc/0601026.

[23] H. Beyer and O. Sarbach, Phys. Rev. D 70, 104004 (2004).

[24] C. Bona, L. Lehner, and C. Palenzuela-Luque, Phys. Rev. D 72, 104009 (2005).

[25] B. Gustafsson, H.-O. Kreiss, and J. Oliger, Timedependent Problems and Difference Methods (John Wiley \& Sons, New York, 1995).

[26] H. R. Beyer, gr-qc/0510097.

[27] M. Alcubierre et al., Phys. Rev. D 62, 124011 (2000).

[28] C. Gundlach and J. M. Martín-García, Phys. Rev. D 70, 044031 (2004).

[29] A.M. Khoklov and I.D. Novikov, Classical Quantum Gravity 19, 827 (2002).

[30] C. Gundlach, documentation for Cactus thorn "Exact," www.cactuscode.org (1998). 